\title{
The benefits of memory control processes in working memory: comparing effects of self-reported and instructed strategy use
}

\author{
Lea M. Bartsch ${ }^{1}$, Alessandra Souza ${ }^{1,2} \&$ Klaus Oberauer ${ }^{1}$ \\ ${ }^{1}$ University of Zurich, ${ }^{2}$ Center for Psychology, Faculty of Psychology and Education \\ Sciences, University of Porto
}

Author Note

We thank Atalia Adank, Natasa Milovanovic, and Dawid Strzelczyk for helping with data collection. The data and the analysis scripts can be accessed in the Open Science Framework (https://osf.io/m2hx3/). K. Oberauer (grant 100014_149193) and A. S. Souza (grant 100019_169302) were supported by grants from the Swiss National Science Foundation. A. S. Souza was additionally supported by national funds from the Portuguese Foundation for Science and Technology (UIB/00050/2020).

Correspondence should be addressed to Lea M. Bartsch, Department of Psychology, Cognitive Psychology Unit, University of Zurich, Binzmuehlestrasse 14/22, 8050 Zurich, Switzerland. E-mail: 1.bartsch@psychologie.uzh.ch. 


\begin{abstract}
Working memory performance is often assumed to benefit from different maintenance control strategies such as rehearsal, refreshing, elaboration, and grouping. In studies assessing strategy self-reports, some strategies were indeed associated with better recall. Nevertheless, experimental studies assessing the effect of instructing maintenance strategies compared to a no-instruction baseline lend no evidence for the effectiveness of these strategies for working memory. Explanations for this contradiction could be that instruction implementation engenders dual-task costs, or that strategy instructions reduce adaptive strategy switching. Across two experiments, we investigated the frequency and variability of strategy use with trial-wise self-reports in serial recall of word lists. Further, we examined potential instruction costs by comparing performance in trials with self-reported vs. instructed use of the same strategies. Self-reported strategy use varied from trial to trial, with elaboration and rehearsal being the most frequent. Self-reported elaboration was correlated with better performance than reading and rehearsal. For the most prevalent strategies - elaboration and rehearsal there were no costs of instructed strategy-implementation. Our results speak against dual-task costs, and for an advantage of adaptively choosing one's own strategy from trial to trial.
\end{abstract}

Keywords: Strategies, Working Memory, Long-term Memory, Elaboration, Rehearsal, Refreshing 
The benefits of memory control processes in working memory: comparing effects of selfreported and instructed strategy use

Working memory (WM) is understood as a capacity-limited store that holds information available for ongoing processing. Maintenance strategies - such as rehearsal, refreshing, different forms of elaboration, as well as grouping - are assumed to control what is retained in WM, with different effects on immediate and delayed recall (Atkinson \& Shiffrin, 1968). Rehearsal refers to the overt or covert repetition of the memoranda to oneself (Baddeley, 1986). Elaboration encompasses processes that enrich the memory representation of an item by activating many aspects of its meaning and by linking it into the pre-existing network of semantic associations (Craik \& Tulving, 1975), as for example the creation of a sentence linking words presented for study, or the use of mental imagery. Refreshing is defined as a domain-general attentional process that is understood as briefly thinking of a stimulus just after it is no longer physically present but while its mental representation is still active (Johnson et al., 2002). Grouping is defined as organizing the memory list into shorter groups of pairs or triplets (Ryan, 1969a).

In some theories of WM, maintenance processes such as rehearsal and refreshing play a key role in explaining people's performance in immediate memory tasks (e.g., Baddeley, 1986; Barrouillet et al., 2011; Cowan, 2005). In other theories, maintenance processes play a more minor role (Nairne, 1990), or none at all (Lewandowsky \& Farrell, 2008; Oberauer et al., 2012). Therefore, finding out which maintenance processes people engage in, and whether they are effective in maintaining information in WM, contributes to evaluating competing theories of WM. This is the aim of the present work.

Studies have shown that in WM tasks, subjects report to engage in these strategies to different degrees (Bailey et al., 2008, 2011; Dunlosky \& Kane, 2007; Morrison et al., 2016; Richardson, 1998). Also, choice of strategy in these studies is correlated with retrieval success 
- some self-reported strategies such as mental imagery, sentence generation, and grouping are associated with better memory than others. Yet, this positive correlation between recall and self-reported strategy use is not sufficient to establish a causal effect of these strategies on memory storage. For instance, the relationship could equally well be explained by having a good representation of the current memory set being a prerequisite for carrying out strategies such as elaboration. Alternatively, participants could be using memory strength to infer the sorts of strategies that they may have used to encode this information. In order to draw causal inferences, we must turn to experimental evidence: Past research has compared performance under different experimental conditions in which the respective strategies were instructed, leading to inconclusive results or even evidence against their benefit (for a review see Oberauer, 2019).

Here we investigate possible explanations for why correlational studies on strategyuse show consistent correlations with performance in typical WM tasks, such as complex or simple span (Bailey, Dunlosky, \& Kane, 2008, 2011; Dunlosky \& Kane, 2007; Richardson, 1998), whereas experimental attempts to improve performance by inducing strategies that were correlated with better performance in the aforementioned studies never succeeded (e.g., Bartsch, Singmann, \& Oberauer, 2018; Jonker \& Macleod, 2015; Souza \& Oberauer, 2018). In the following, we briefly summarize both the correlational as well as experimental evidence for each strategy.

\section{Self-Reported Use of Memory Control Processes}

In past self-report studies, strategy use has been mostly assessed by asking participants at the end of the experiment to indicate for each trial - presented to them again - which strategy they used in that trial: reading, rehearsal, sentence generation, imagery, grouping, or something else. Rehearsal is generally reported most frequently, occurring in ca. $33-50 \%$ of trials of simple and complex span tasks; followed by reading (21-43\%), mental imagery (6- 
$14 \%$ ), grouping (5-13\%), and sentence generation (2-13\%; see Bailey, Dunlosky, \& Hertzog, 2009; Bailey et al., 2008, 2011 for details). Span performance is significantly higher in trials in which individuals report to engage in mental imagery and sentence generation compared to merely reading each word as it is presented, or engaging in rehearsal (Bailey et al., 2008, 2009, 2011; McNamara \& Scott, 2001).

Given this correlational evidence on the effectiveness of some of these strategies, it seems plausible that either (a) increasing the time interleaving the memoranda in a WM task, or (additionally) (b) instructing a specific strategy in some trials, would allow any individual to engage in effective strategies more often, or to switch from a less effortful strategy (e.g., reading) to one that requires more processing, but which leads to a larger mnemonic benefit (e.g., sentence generation). In the following, the experimental evidence for this hypothesis and the role of each strategy is briefly summarized.

\section{Experimental Evidence for a Beneficial Role of Maintenance Strategies in WM}

To measure the occurrence of rehearsal, some previous studies have employed overt rehearsal protocols: participants are instructed to carry out their rehearsals overtly and the experimenter records them for analysis. This method permits the online evaluation of rehearsal as it unfolds in the WM task. Tan and Ward (2008) used an overt rehearsal protocol and observed a correlation between the length of cumulative rehearsal - i.e., looping through memory items in their order of presentation - and the benefits of increasing free time in a serial recall task. These results suggest that participants benefit from free time because they can rehearse the memoranda in the pauses between items.

Experimental work, however, has challenged the causal role of cumulative rehearsal for promoting memory benefits: One early study tested the effect of practicing cumulative articulatory rehearsal on performance in the complex-span paradigm (Turley-Ames \& Whitfield, 2003). The trained group showed better performance when encoding was self- 
paced, yet this was because they took more time to encode the items. When the time to encode each item was controlled by the experimenter, the advantage of the trained group disappeared.

Using the overt rehearsal protocol, Souza and Oberauer $(2018,2020)$ showed that increasing the number and length of cumulative rehearsals during free time via instructions did not result in better performance compared to conditions without instruction (free rehearsal) in simple span as well as complex span tasks. These results cannot be explained by rehearsal being already maximally beneficial in the free rehearsal baseline. In one experiment, participants were instructed to read aloud during the free-time intervals either the spontaneous rehearsals performed by a yoked participant in the free-rehearsal condition, or irrelevant syllabi ("bababa"). Reading the rehearsals of another participant should facilitate rehearsal by even removing the necessity to retrieve memory items, whereas irrelevant articulation impedes rehearsal. Nevertheless, performance in these two conditions did not differ (Souza \& Oberauer, 2018).

Elaboration (which encompasses sentence generation and imagery) is the second most commonly reported strategy. Recent studies investigating the effect of experimentally instructing elaboration in WM found no evidence for a WM benefit, although elaboration promoted long-term memory (LTM) (Bartsch et al., 2018, 2019; Bartsch \& Oberauer, 2021; Jonker \& Macleod, 2015). In the instructed elaboration condition, WM performance was never better, and in most cases worse, than in a baseline condition in which participants were free to do whatever they wanted.

Refreshing is a more recent addition to the list of maintenance strategies (see Camos et al., 2018 for a review). Participants hardly ever report spontaneously engaging in refreshing (Loaiza \& Lavilla, 2021). Refreshing, however, has been argued to improve WM (Souza et al., 2015, 2018; Souza \& Oberauer, 2017; Vergauwe \& Langerock, 2017) as well as longterm memory (Camos \& Portrat, 2015; Loaiza \& Mccabe, 2012). Studies explicitly guiding 
attention to individual WM elements via retro-cues during the retention interval improved performance for tests of the cued items, and performance increased with the number of guided refreshing steps (Souza et al., 2018). Nevertheless, instructing people to refresh a memory set did not lead to any WM or LTM benefits in comparison to a free baseline (Bartsch et al., 2018).

Participants also report grouping in a small proportion of trials (5-13\%). Experimental evidence suggests that serial recall performance is higher in case the items are presented in groups of threes - operationalized through the insertion of temporal gaps at the group boundaries - compared to ungrouped presentation (e.g., Broadbent \& Broadbent, 1981; Hartley, Hurlstone, \& Hitch, 2016; Hitch, Burgess, Towse, \& Culpin, 1996; Liu \& Caplan, 2020; Ryan, 1969a, 1969b). Yet, imposing a temporal gap through the rhythm of the stimuli is not the same as participants trying to create groups of items presented at a constant pace. Early work indicated that performance in a condition with merely instructed grouping was numerically better than an ungrouped condition, but not significantly different (Ryan, 1969a, Exp 1). More recent work indicates that instructed grouping - when the list presentation is ungrouped - shows similar effects to those observed under temporal grouping on immediate memory performance (Farrell, 2008).

A recent study by Barrouillet et al. (Barrouillet et al., 2020) combined instructions of rehearsal and refreshing for different subsets of the memory list - a procedure they termed the maxispan. In the basic procedure, part of the memory list was presented in blue (3-5 letters) and the remaining items ( 1 to 6 letters) were presented in black font. Participants in the maxispan condition were instructed to rehearse the blue letters and mentally maintain the black letters; whereas participants in the control condition did not receive instructions regarding the colors. The maxispan procedure yielded higher spans when participants were instructed to rehearse 4 to 5 letters, especially when these were followed by large sets of black letters. This study suggests that instructing rehearsal could be beneficial particularly when 
large lists are presented (lists larger than six items) and when subsets of the list are clearly segregated (using different colors or presentation modalities). Yet, given that this study did not include control conditions in which subsets of the list were not grouped, or in which rehearsal (or refreshing) of all letters was instructed, it is difficult to separate what was the contribution of grouping, or whether this effect is due to rehearsal of only a subset of the list, refreshing of only a subset of the list, or both. Furthermore, the combination of rehearsal and refreshing is not something that participants report doing on their own (and notice that their baseline is a free-strategy condition). Hence, it is unlikely to account for the conflicting results between the correlational and experimental studies on strategy use.

Taken together, the maintenance strategies that some prominent theories of WM assume to be effective (i.e., rehearsal and refreshing), or that self-report studies suggest to be effective (i.e., elaboration), did not show a consistent benefit in experimental studies in which these strategies were instructed. This led to questioning their beneficial role for WM in general.

\section{Hypotheses on Discrepancies Between Self-report and Experimental Instruction Studies}

On the one hand, correlational studies indicate a relation between engaging in certain maintenance strategies and better recall from WM whereas, on the other hand, most experimental studies that manipulated the engagement in these strategies have observed no evidence that they improve memory. Why do these two sets of studies yield conflicting evidence?

We can think of two possible explanations. First, experimentally instructing a strategy of interest requires participants to maintain and carry out the strategy as instructed, which can function as a secondary task, thereby creating a dual-task cost on WM and counteracting any potential benefits of the instructed strategy. Given that previous studies have only compared performance between the strategy instruction condition against a no-instruction baseline 
(which likely involves a mixture of different strategies), their null findings may reflect a mixture of a benefit of the strategy and a cost of the additional task-set to be maintained in mind. To uncover a potential dual-task cost of instructed strategies, one needs to compare performance in conditions with instructed and self-selected strategy use. One prediction from this hypothesis is that dual-task costs should be larger particularly for those strategies that are more cognitively demanding. Previous research has shown that elaboration is fairly demanding, rehearsal is modestly demanding, and reading is minimally demanding (NavehBenjamin \& Jonides, 1984; Thalmann et al., 2019). Refreshing has also been conceptualized as a fairly demanding strategy (Camos et al., 2018). Accordingly, we should expect instructions to elaborate and refresh to be more costly to implement, followed by rehearsal, and lastly by reading.

Second, self-report studies show that individuals spontaneously apply a mixture of various memory strategies over the course of an experiment. This may indicate that they adaptively shift from one strategy to another depending on the memoranda. Studies in which participants are instructed to use one strategy throughout prevent such adaptive switches, thereby resulting in worse performance across all trials than in a baseline condition in which people can freely switch between strategies. If this is the case, the difference between performance in instructed trials and in trials where people use the same strategy in the free baseline condition would become most apparent for the strategies that are rarely chosen, because in those cases, the instructed strategy is not the best for the majority of trials.

\section{The Present Experiments}

The goal of the present study was to investigate the effectiveness of WM strategies for maintenance. To resolve the ambiguity from previous research on this question, we asked whether instructions to engage in a certain strategy alter memory performance compared to when participants indicate to have engaged in the same strategy spontaneously. To that end 
we were interested in whether - within a person rather than across studies - performance in trials of self-reported use of a given strategy differs from performance of that same participant when this strategy was instructed. If performance is better when participants spontaneously engage in a strategy, this may indicate that instructed strategy-use does not produce a benefit in experimental studies because maintaining and implementing the instruction imposes a cost, or because it precludes natural variability and adaptive shifting between strategies.

Across two experiments, we invited participants into the lab on two separate days to complete two sessions of a verbal serial-recall task in which the memoranda were interleaved with generous free time - providing ample opportunity to engage in any of the above candidate strategies. In the first session, participants were free to engage in any strategy they wanted, and their chosen strategies were measured with strategy self-reports after recall in each trial. This procedure allowed us to get an insight into the trial-to-trial variability or stability of using the different strategies. Strategy reports after each trial are preferable over global strategy reports or retrospective reports collected at the end of the experiment because retrospective reports could be distorted by participants mis-remembering their own strategies. Importantly, strategy reports after each trial have been shown previously to have minimal reactive effects, with the reporting of a strategy on one trial having little influence on strategy selection or immediate serial recall performance on subsequent trials (Dunlosky \& Kane, 2007).

In the second session, participants were instructed to use one of the memory strategies for mini-blocks of four trials. After recalling all items of each trial, they were to indicate whether they had complied with the instruction, and if not, what they had done instead. We kept the order of sessions constant to avoid any influence of the instructions of strategies on the pattern of strategies self-chosen by the participants. We discuss possible confounding effects of this choice such as fatigue and practice effects in the General Discussion. Both sessions in Experiment 1 further entailed a delayed free recall test for all the items presented 
during the session in order to measure the influence of the WM strategy applied for the formation of LTM representations.

Experiment 2 implemented the same design and task, with one difference: Whereas in Experiment 1 we used a large open set of words as memory items, in Experiment 2 we used a small, closed set of memory items which were repeated in random order from trial to trial. The repeated use of the same small set of words makes it harder to distinguish successive trials from each other, and increases the demand to remember in which particular order the words have been presented in the current trial. This increases proactive interference between trials in episodic LTM, and therefore, participants in Experiment 2 were less able to rely on episodic memory for immediate serial recall. The comparison between Experiment 2 and Experiment 1 enables us to assess whether participants adapted their strategy use to the fact that they could rely less on episodic LTM to complete the WM task. We expected that an adaptive strategy choice should lead to a reduction of elaborative strategies in Experiment 2, because elaboration is a particularly effective strategy for improving episodic memory.

\section{General Method}

\section{Participants}

For Experiments 1 and 2, we recruited two independent samples of 30 students (E1: 20 females; mean age $=23.47$; E2: 26 females; mean age $=22.13$ ) from the University of Zurich. We chose the sample size because it is sufficient to detect medium to large effects in withinsubjects designs. The use of Bayesian statistics means that the sample size could have been increased in case of ambiguous evidence (Rouder, 2014), but this was not necessary.

In both studies, participants were compensated with either 45 Swiss Francs (about 45 USD) or partial course credit for completing two sessions, with the first lasting one and the second lasting two hours. Participants signed an informed-consent form before the start of the experiment and were debriefed at the end. The studies were carried out in agreement with the 
rules of the Ethics Committee of the Faculty of Arts and Sciences of the University of Zurich and did not require special approval.

\section{Materials and Procedure}

In both experiments, lists of six nouns were studied for a forward serial recall test. The stimuli were drawn from a pool of 1182 German abstract and concrete nouns (591 each).

Concrete nouns had a mean imageability rating of 5.65 (range $=4.54-6.89$; on a 7-point scale) and abstract nouns of 3.16 (range $=1.35-4-45)$. The nouns were between three and ten letters long and had a mean frequency of 58.58/million (Vo et al., 2009). In both sessions of Experiment 1, stimuli were drawn without replacement from the large pool of abstract and concrete nouns, and within a given trial all items were either abstract or concrete. For each session of Experiment 2, six abstract and six concrete nouns were drawn from the large pool once for each subject, and stimuli for each trial in that session were randomly drawn only from this small pool of 12 words irrespective of concreteness. ${ }^{1}$

The sequence of an experimental trial is illustrated in Figure 1. Prior to completing the WM task, subjects were informed about the possible memory strategies people typically use during WM tasks, specifically about mere reading, rehearsal, refreshing, mental imagery, sentence generation, and grouping. Across four practice trials, they were familiarized with the task.

A fixation cross cued the beginning of a trial for $1000 \mathrm{~ms}$. Thereafter, six words were presented sequentially for $500 \mathrm{~ms}$ with a $4500 \mathrm{~ms}$ blank inter-item-interval. The memoranda $($ font size $=24 \mathrm{pt})$ were presented in boxes (size $=200$ pixels $)$ equidistantly arranged on an

\footnotetext{
${ }^{1}$ For Experiment 1, we included word concreteness as a factor in the analysis on performance, but that factor had only a main effect, without entering into any interactions, and therefore we decided to drop it for Experiment 2 and present participants with mixed lists of abstract and concrete words for simplicity. The interested reader can find a Figure and the analysis of the mean proportion of trials in which each strategy was reported separated by concreteness in the Supplementary Materials (Figure S9).
} 
invisible circle centered on the screen in clockwise order, starting at the top box. After the blank following the sixth word, the WM test followed: A box appeared in the middle of the screen prompting subjects to type in the words one-by-one in forward serial order.

Participants did not receive feedback regarding memory accuracy after the WM trials.

\section{Figure 1}

Panel A. Illustration of the Working Memory Paradigm. A) Participants were shown a list of six words sequentially across different boxes on the screen and tested with typed recall in forward serial order. B) In Session 1, after recall of each list subjects were prompted to indicate which of the memory strategies, they had engaged in. C) In Session 2, a prompt like the one depicted in the panel instructed subjects which strategy to use during the upcoming trial. Here one example condition (reading) is depicted; instructions of the other strategies in Table 1 used an analogous screen display. After recall participants were asked whether they had followed this instruction during the past trial.

A
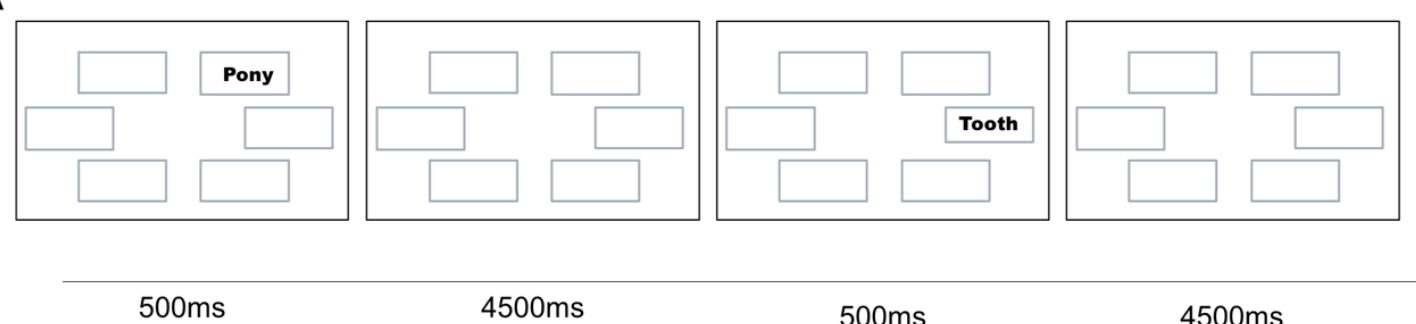

$4500 \mathrm{~ms}$

$500 \mathrm{~ms}$

$4500 \mathrm{~ms}$
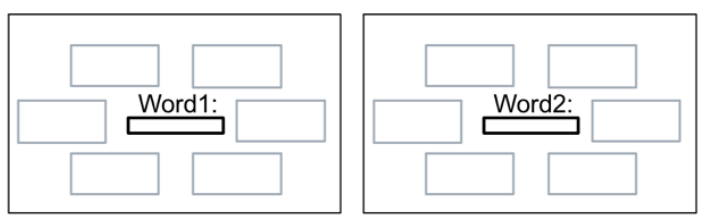

B

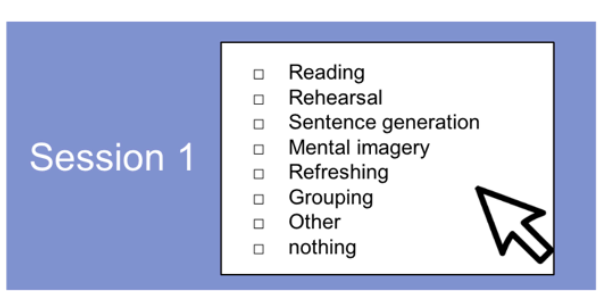

C

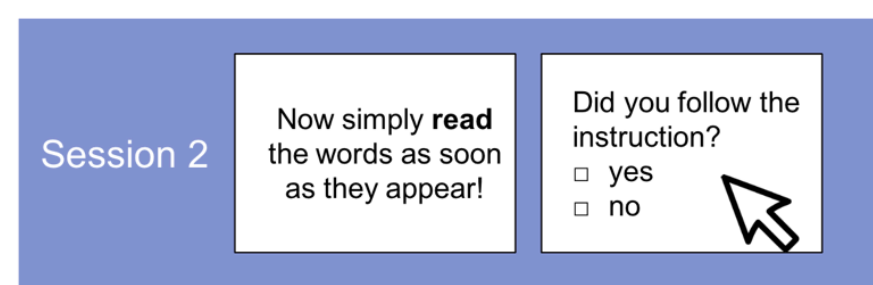

In Session 1, after recalling all six items, subjects were prompted to indicate which strategies they had engaged in during the past trial by clicking on the respective option. The participants were allowed to choose only one strategy per trial. The options were (with the original label in German): (1) reading (lesen), (2) rehearsal (wiederholen), (3) refreshing, 
described as directing attention to the memoranda (Aufmerksamkeit auf Gedächtnisinhalte legen), (4) mental imagery (bildlich vorstellen), (5) sentence generation (Satz generieren), (6) grouping (gruppieren), (7) other (eine andere Strategie), and (8) nothing (nichts). The strategy prompts and the strategy descriptions provided at the beginning of the experiment were adapted from Dunlosky and Kane (2007), except for the refreshing instruction, which was based on descriptions provided to us by 12 experts in the field of refreshing ${ }^{2}$. The (translated) general descriptions can be found in

Table 1.

\section{Table 1}

Strategy Descriptions and Instructions used in Session 1 and 2 of Experiment 1 and 2.

\begin{tabular}{|c|c|c|}
\hline Strategy & Description provided in Session 1 & Mini-block instruction in Session 2 \\
\hline reading & $\begin{array}{l}\text { You just read the words as they } \\
\text { appeared on the screen. }\end{array}$ & $\begin{array}{l}\text { Simply read the words as soon as they } \\
\text { appear on the screen. }\end{array}$ \\
\hline rehearsal & $\begin{array}{l}\text { You repeat the words as often as } \\
\text { possible in your head or out loud. }\end{array}$ & $\begin{array}{l}\text { Repeat the words in your head or aloud } \\
\text { as many times as possible. }\end{array}$ \\
\hline refreshing & $\begin{array}{l}\text { You direct your attention back to all } \\
\text { the words presented so far. You do } \\
\text { not articulate the words, but only } \\
\text { think quickly and briefly about each } \\
\text { word. }\end{array}$ & $\begin{array}{l}\text { Direct your attention back to all the } \\
\text { words presented so far. You should not } \\
\text { articulate the words, but just think } \\
\text { quickly and briefly about each word. }\end{array}$ \\
\hline mental imagery & $\begin{array}{l}\text { You mentally imagine the words in } \\
\text { one picture. }\end{array}$ & $\begin{array}{l}\text { Imagine the words in one mental } \\
\text { picture. }\end{array}$ \\
\hline sentence generation & $\begin{array}{l}\text { You form a sentence to link the } \\
\text { words together. }\end{array}$ & $\begin{array}{l}\text { Form a sentence using the words in } \\
\text { order to link the words together. }\end{array}$ \\
\hline grouping & $\begin{array}{l}\text { You memorize the words in groups, } \\
\text { for example, always in groups of } 2 \text { or } \\
\text { memorize the first half of the list } \\
\text { together and the second half together. }\end{array}$ & $\begin{array}{l}\text { Memorize the words in groups, for } \\
\text { example always in groups of } 2 \text { or the } \\
\text { first half of the list together and the } \\
\text { second half together. }\end{array}$ \\
\hline
\end{tabular}

In Session 2, prior to the start of the sequential presentation of memoranda, subjects

were instructed to use one of the six memory control processes for the upcoming mini-block of four trials (see Table 1 for the mini-block instructions of the strategies). After recalling the

\footnotetext{
${ }^{2}$ We thank the attendees of the workshop: "The Crossroads of Attention in Working Memory: Consolidation, Refreshing, \& Removal" for their descriptions provided to us anonymously.
} 
six words, the trials in Session 2 ended with a screen asking subjects whether they had complied with the instructions, and if not, they were prompted to indicate which strategies they used instead, using the same eight-alternative questionnaire as in Session 1.

In Session 1 there were 60 trials of the WM task, and Session 2 comprised 24 trials per strategy for a total of 144 trials. For Experiment 1, half of the trials of each session consisted of concrete and the other half of abstract lists. In Experiment 2, the stimuli in each session were drawn randomly from the pool of six concrete and abstract nouns, thereby concreteness varied within trials and was not considered as an experimental variable.

After the end of the WM task in each session of Experiment 1, an unrelated distracter task followed in which participants had to indicate the correctness of visually presented math equations (e.g., $9 \times 8=72$ ) for 2 minutes. After that, there was a typed delayed free recall memory test, wherein the participants were asked to recall as many memory items from the WM task as possible. This test served to assess the effect of each WM strategy on episodic LTM. Participants were made aware of the delayed memory test before the start of the experiment.

\section{Data Analysis}

The dependent variables of interest were: (a) frequency of strategy self-reports in Session 1, and (b) serial recall accuracy (i.e., the proportion of words correctly recalled using strict correct in-position scoring) as a function of self-reported and instructed WM strategy. Further, we also investigated the trial-to-trial variability in self-reported strategy use by examining the transition probabilities of reporting one strategy given the strategy reported in the previous trial. We analyzed the data using Bayesian mixed effect models (LME) using the ImBF function implemented in the BayesFactor package (Morey et al., 2015) in R (R Core Team, 2017). With this we calculated Bayes Factors (BFs) which represent the strength of evidence for a specified model (M1) against a Null or reduced model (M0). For instance, we 
can calculate the evidence for the effect of WM strategy $\left(\mathrm{BF}_{10}\right)$ by comparing the evidence for a model including this factor against an intercept-only model that serves as the null model. Additionally, we can calculate evidence against an effect of $\mathrm{WM}$ strategy $\left(\mathrm{BF}_{01}\right)$, where $\mathrm{BF}_{01}$ $=1 / \mathrm{BF}_{10} . \mathrm{A} \mathrm{BF}_{10}$ larger than 1 gives evidence for an effect, $\mathrm{a} \mathrm{BF}_{10}$ lower than 1 provides evidence against an effect and hence evidence for the null hypothesis. $\mathrm{A} \mathrm{BF}_{10}$ of 10 indicates that the data are 10 times more likely under the alternative hypothesis than under the null hypothesis. Usually, BFs below 3 are regarded as anecdotal evidence and BFs $>3$ are regarded as providing substantial evidence for one hypothesis over the other (Kass \& Raftery, 1995). All models included a random intercept for participant, and random slopes over participants for the effects of the variables manipulated within-subjects (Barr et al., 2013).

\section{Results}

In the following, we first report the results of both experiments jointly in light of two research questions about strategy use: (1) Which strategies do subjects report to engage in spontaneously? (2) Do subjects comply with strategy instructions? We first address which strategies were reported in Experiment 1 and 2, and whether subjects are consistent in the memory strategies they use. We then shortly examine the compliance rate across both experiments.

Then we turn to the performance in the delayed memory test of Experiment 1 to answer the question (3): Do the strategies impact LTM performance? This allowed us to assess if strategy effects are mediated by the creation and use of LTM representations. Next, we present performance the immediate memory test of Experiments 1 and 2 to answer our main research question: (4) Do strategies impact WM? By answering these questions, we test our two main hypotheses: (a) Strategy instruction produces a general dual-task cost in WM that prevents their benefits from being measured; and (b) strategy instruction prevents 
adaptive switching between strategies. We do so by comparing individuals' performance in trials of self-reported vs. instructed strategy use.

All data and analysis scripts can be accessed on the Open Science Framework (https://osf.io/m2hx3).

\section{What Strategies do Subjects Spontaneously Use During Free Time?}

\section{Strategy Self-Reports}

The mean proportion of trials in which each strategy was reported in Experiments 1 (Open Pool) and 2 (Closed Pool) is shown in Figure 2. This variable was calculated by first computing the mean proportion of trials in which each strategy was reported by each subject, and then averaging across all subjects. As a first step, we were interested in the overall strategy occurrence and how they rank from most to least frequent.

When subjects were able to also rely on episodic LTM traces of the memoranda during the WM task - because the memoranda only occurred once in the experiment, as it was the case for the open pool of stimuli in Experiment 1 - the most reported strategies were sentence generation, mental imagery, rehearsal, and grouping, in that order. Other strategies, such as refreshing or reading, were very rarely reported.

In Experiment 1, concreteness increased the probability of choosing mental imagery and grouping (see Figure S9 in the online supplementary material for details). Rehearsal and reading were more likely to be chosen in abstract than concrete trials. We turn to the question of whether concreteness also affected the effectiveness of the instructed strategies below. 


\section{Figure 2}

Mean Proportion of Self-Reported Strategies in Session 1 of Experiment 1 (Top Panel) and in Experiment 2 (Bottom Panel). Experiments 1 and 2 Differ in Terms of the Pool of Words Used (Open vs. Closed). Error Bars Reflect the Standard Error of the Mean.

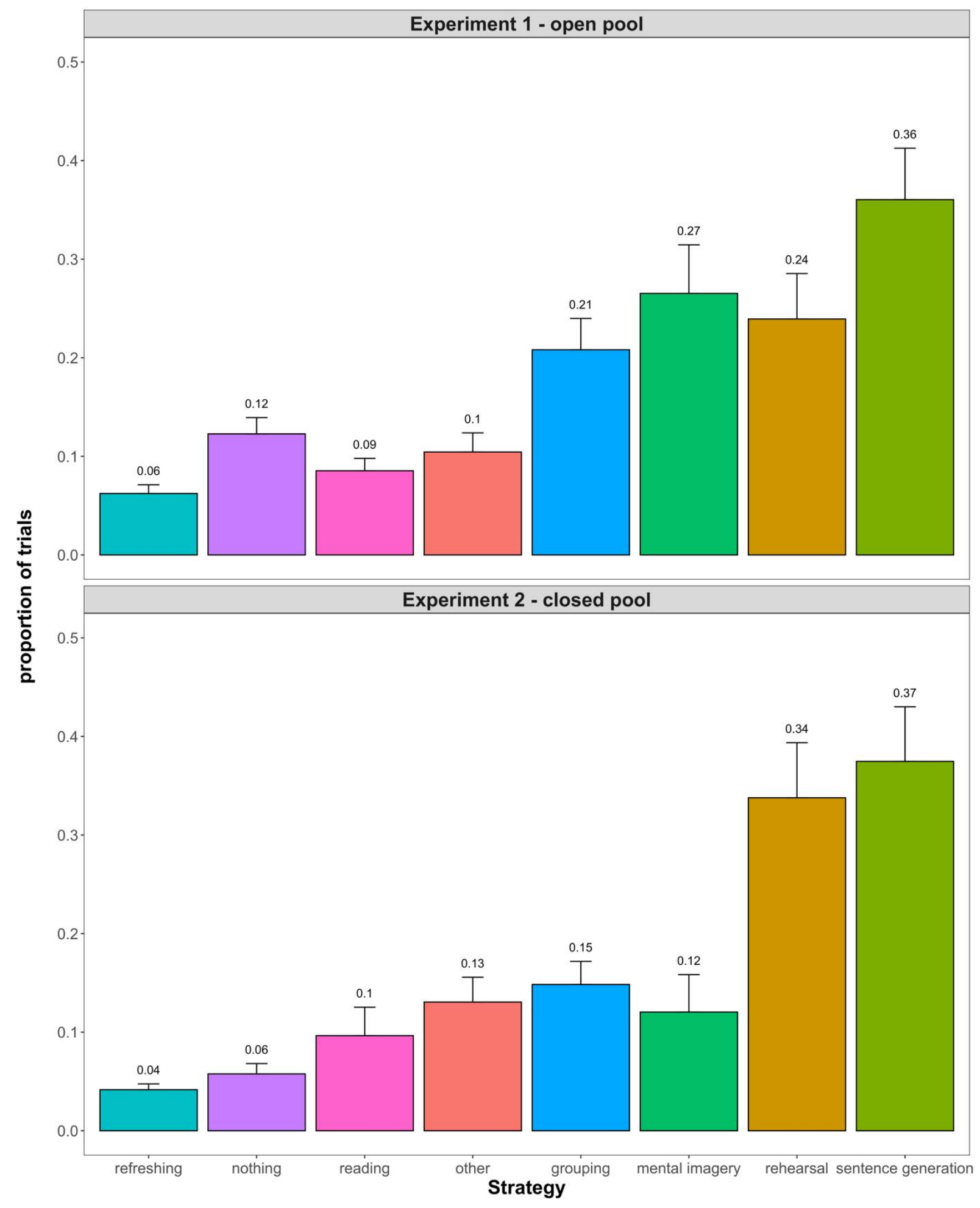


Strategy use changed when subjects could rely less on episodic LTM traces due to a build-up of proactive interference, as was the case for the closed set of stimuli in Experiment 2: Specifically, there was a strong drop in the use of mental imagery and grouping, as well as an increase in rehearsal. Yet, sentence generation remained the most used strategy. The rare reporting of refreshing and reading persisted in Experiment 2.

\section{Strategy Switches Between Trials}

Figure 3 shows the transition probabilities of reporting one strategy given the strategy reported in the previous trial in Experiments 1 and 2. This figure illustrates the trial-to-trial variability in strategy use. In both experiments, subjects were generally more likely to switch than to stay with a certain strategy, with a mean switching probability of $77.95 \%$ (SD = $11.72)$ and $72.54 \%(\mathrm{SD}=17.00)$ for Experiments 1 and 2, respectively.

Trial-to-trial variability in strategy use might reflect changes induced by task exposition such as learning or fatigue. To investigate this, we plotted the proportion of selfreported strategies over trials in Figure 4. It shows that in Experiment 1 there is no sign that more effortful strategies like sentence generation are chosen less often towards the end of a session. On the contrary, in Experiment 2, the subjects seem to learn that sentence generation is a beneficial strategy, leading to a trade off in choosing it over rehearsal as a strategy.

Table 2 shows the descriptive probabilities of switching to a different strategy than the one used in the previous trial for each strategy separately ${ }^{3}$. Although overall, participants were more likely to switch than to stay, when we consider the individual transitions between one strategy to another, participants were more likely to remain with the same strategy than to

${ }^{3}$ The values consider how often a specific strategy was chosen in the first place: The overall probability to switch is calculated as the number of switches divided by the total number of times the strategy switched away from was chosen. We did this hierarchically, meaning we computed the switching probabilities for each individual first, and only then these probabilities were averaged across individuals. This gives us the mean probability of switches across the entire experiment. 
transition to any particular other strategy. One can see this by examining the width of the connections in Figure 3: particularly for rehearsal, sentence generation, and mental imagery, subjects were more likely to continue using this strategy from one trial to the next compared to switching to any other single option.

Trial-to-trial variability in strategy use might reflect changes induced by task exposition such as learning or fatigue. To investigate this, we plotted the proportion of selfreported strategies over trials in Figure 4. It shows that in Experiment 1 there is no sign that more effortful strategies like sentence generation are chosen less often towards the end of a session. On the contrary, in Experiment 2, the subjects seem to learn that sentence generation is a beneficial strategy, leading to a trade off in choosing it over rehearsal as a strategy.

\section{Table 2}

Mean probabilities to switch away from the process of the previous trial for each of the memory control process in Experiment 1 (open pool) and Experiment 2 (closed pool) separately. The standard deviation is shown in brackets.

\begin{tabular}{llc}
\hline $\begin{array}{l}\text { process in } \\
\text { previous trial }\end{array}$ & pool & $\begin{array}{c}\text { mean }(\mathrm{SD}) \text { probability } \\
\text { of switching process }\end{array}$ \\
\hline \multirow{2}{*}{ read } & open & $0.87(0.17)$ \\
& closed & $0.85(0.20)$ \\
rehearse & open & $0.68(0.30)$ \\
& closed & $0.54(0.31)$ \\
refreshing & open & $0.97(0.15)$ \\
& closed & $0.95(0.08)$ \\
mental imagery & open & $0.71(0.24)$ \\
\multirow{2}{*}{ sentence generation } & closed & $0.83(0.28)$ \\
& open & $0.60(0.29)$ \\
grouping & closed & $0.47(0.32)$ \\
& open & $0.76(0.26)$ \\
other & closed & $0.69(0.25)$ \\
& open & $0.86(0.25)$ \\
\hline
\end{tabular}




\section{Figure 3}

Chord Diagram of the Proportion of Transitions Between Self-Reported Strategies Across Trials. The Links Represent the Transitions from the Strategy Reported in Trial n-1 at the Top to the Strategy Reported in Trial $n$ at the Bottom. The Link Width Represents Transition Frequency. The Total Length of the Strategy Sector at the Bottom Represents the Total Strength of the Connections to this Strategy (e.g., Fewer Connections to Refreshing and Reading). Panels A and B Show the Data from Session 1 of Experiment 1 (Open Pool) and Experiment 2 (Closed Pool), Respectively.
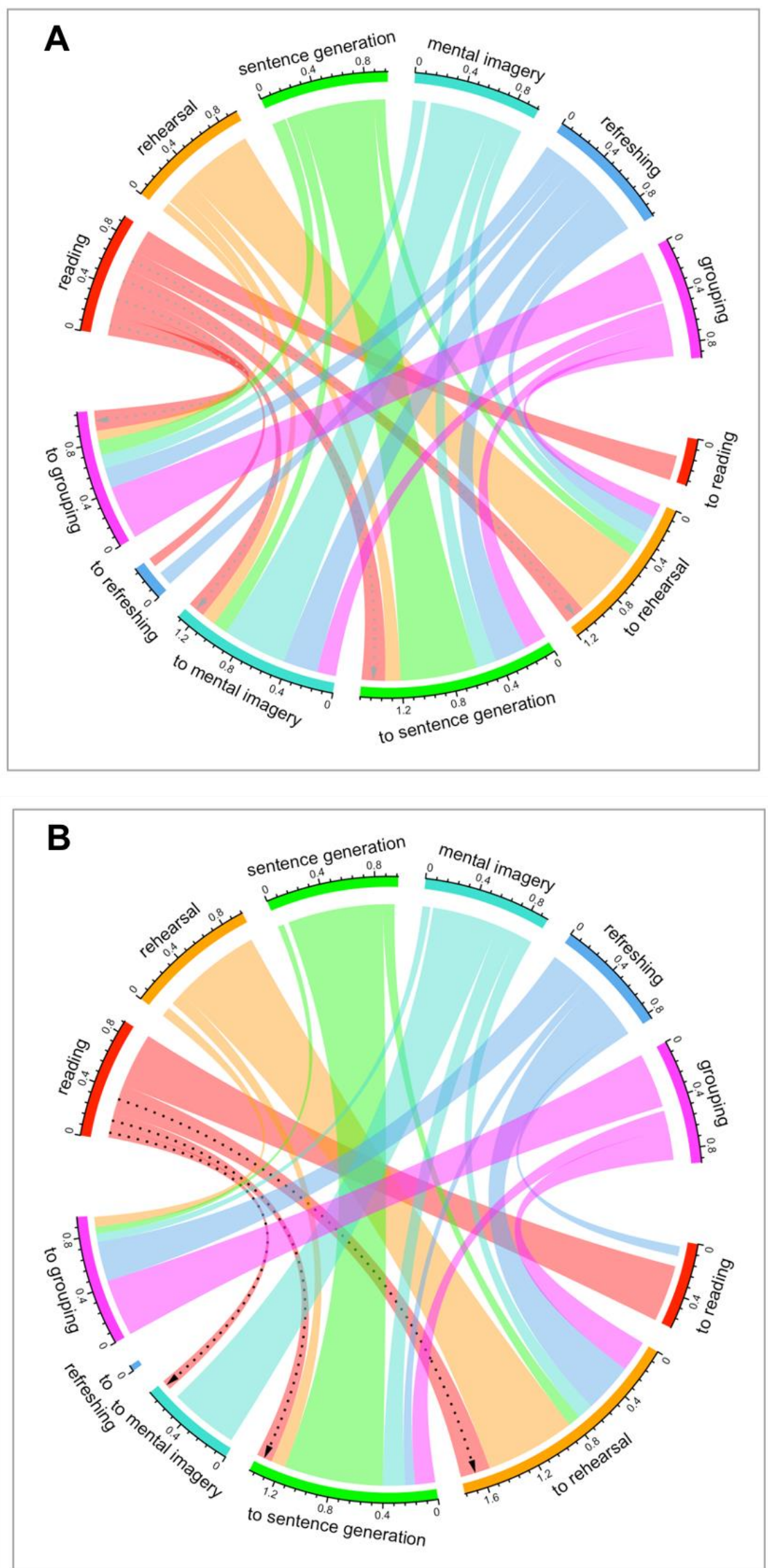


\section{Figure 4}

Proportion of reported strategies as a function of trial in Experiment 1 (Panel A) and Experiment 2 (Panel B). The grey bands represent the smoothed conditional means derived from Local Polynomial Regression Fitting (loess function).

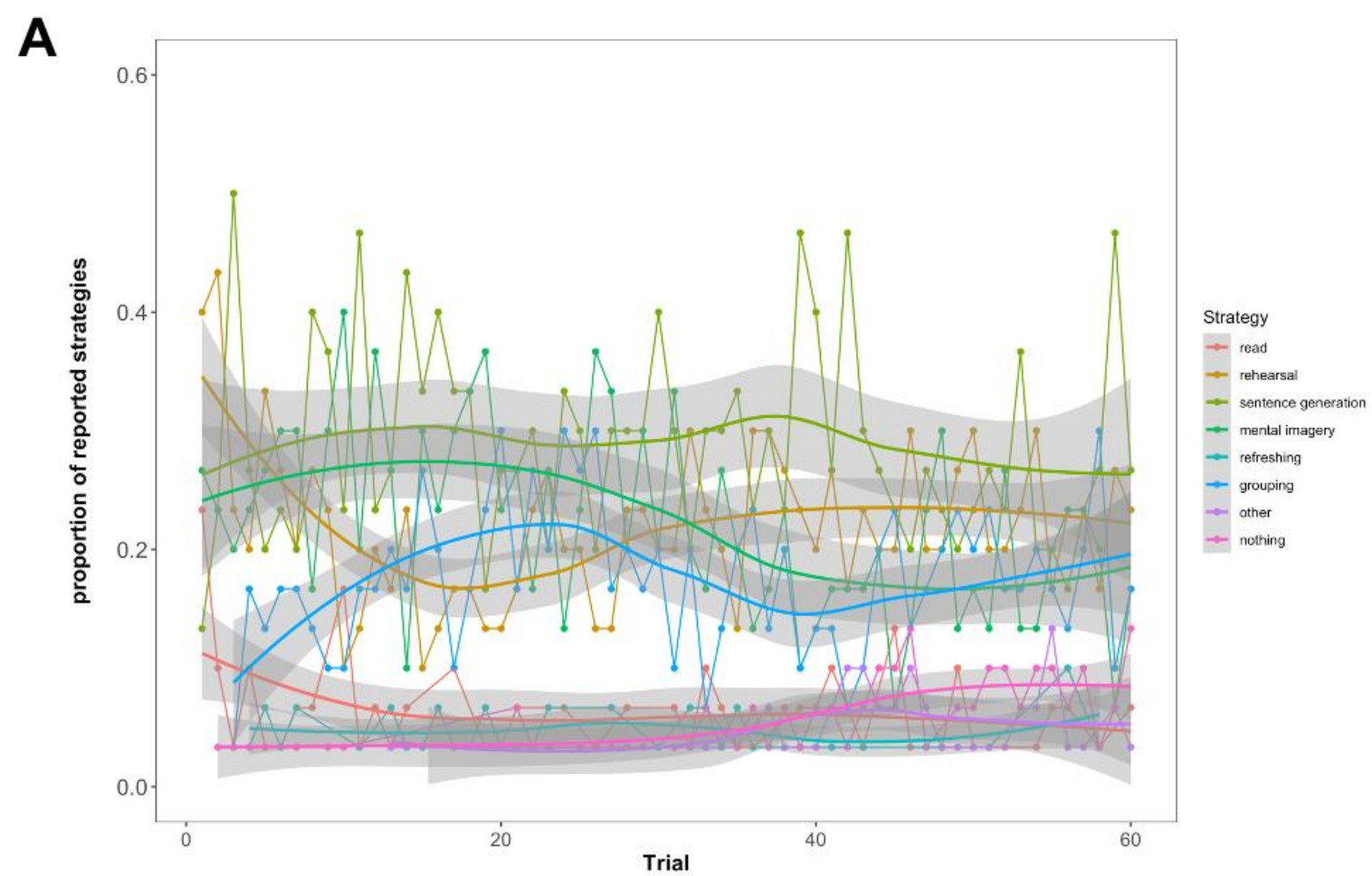

B

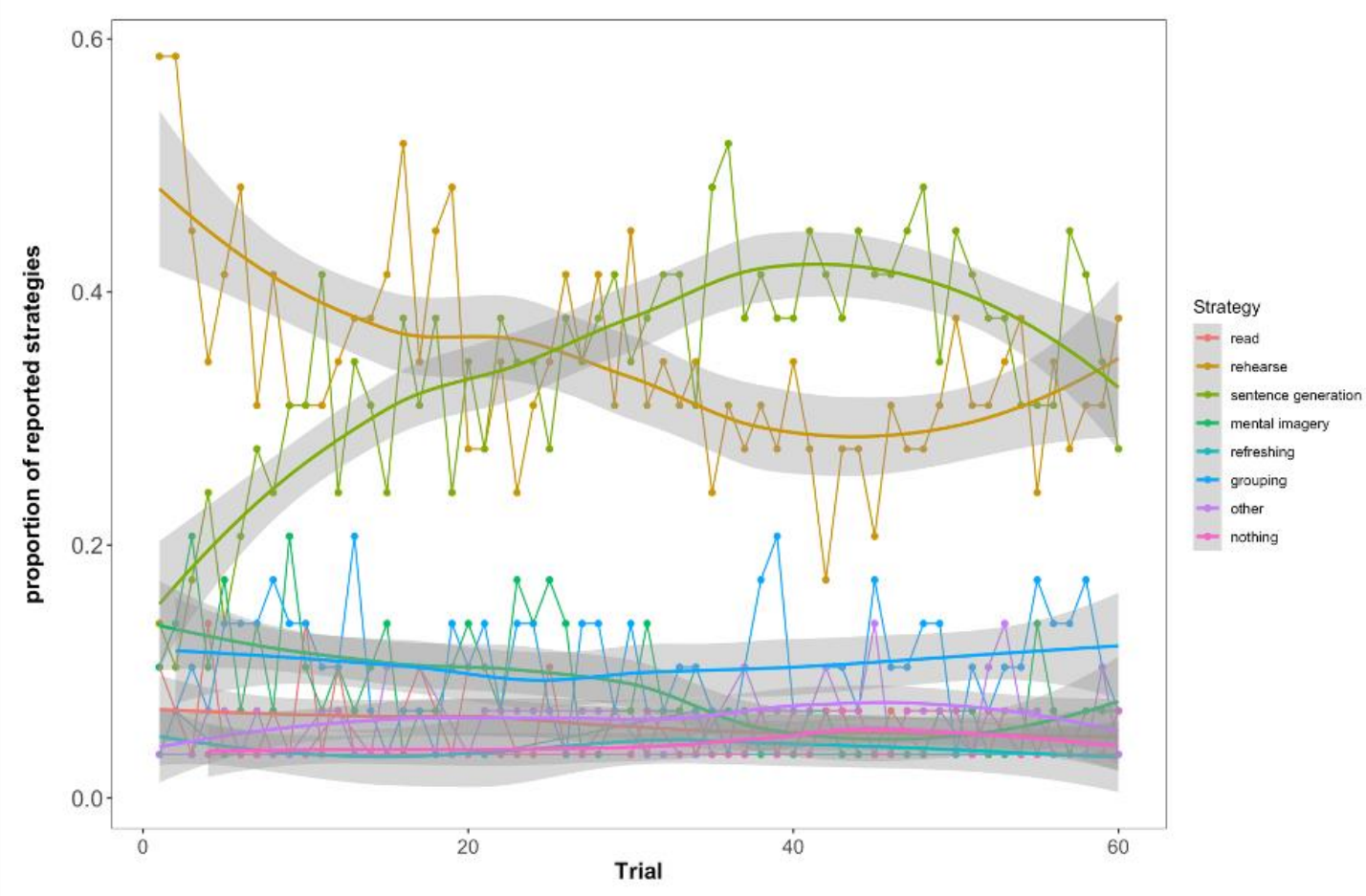




\section{Do Subjects Comply with Strategy Instructions?}

One critical issue in instructing a strategy is to measure compliance with strategy use. In session 2, we asked participants after each instructed-strategy trial whether they did in fact apply the memory control process instructed to them, and if not, what they were doing instead. Subjects indicated to comply with instructions in $84.39 \%$ of the trials $(\mathrm{SD}=24.22)$ in Experiment 1 and $94.37 \%(\mathrm{SD}=18.16)$ in Experiment 2. The Bayesian linear mixed effects model revealed evidence against a difference in compliance across instructed strategies in Experiment $1\left(\mathrm{BF}_{10}=0.0002\right)$. In Experiment 2 there was anecdotal evidence for a main effect of instructed strategy $\left(\mathrm{BF}_{10}=2.64\right)$. Follow-up analyses revealed that compliance was lower when the instruction was to implement refreshing $(\mathrm{M}=89.58 \%$; $\mathrm{SD}=15.58)$ compared to rehearsal $\left(\mathrm{M}=96.04 \% ; \mathrm{SD}=12.44 ; \mathrm{BF}_{10}=1.88,\right)$, sentence generation $(\mathrm{M}=95.20 \% ; \mathrm{SD}=$ 13.31; $\left.\mathrm{BF}_{10}=2.01,\right)$, and grouping $\left(\mathrm{M}=96.66 \% ; \mathrm{SD}=12.39 ; \mathrm{BF}_{10}=3.61\right)$.

In sum, self-reported compliance was very high in both experiments; in the recall performance analyses, only compliance trials were included.

\section{Interim Discussion: Strategy Use}

As a first step, we were interested in which strategies subjects spontaneously report using during a long free time interval between individual memory items. We have shown that subjects engage in some strategies more frequently than others: across both experiments, sentence generation and rehearsal appear as frequent choices, replicating previous findings from self-report studies (Bailey et al., 2011; Dunlosky \& Kane, 2007) - although most previous studies showed more rehearsal and less elaboration. This difference can easily be explained by previous studies giving less time in-between items than we did here. In Experiment 1, in which memoranda were unique in every trial, mental imagery and grouping were also frequent choices. Others, like refreshing were rarely or never reported (see also Loaiza \& Lavilla, 2021). Further, concreteness of the words affected the choice of strategies, 
with subjects choosing to engage in mental imagery and grouping more in case of concrete words. The effect on choosing imagery was expected, because concrete words lend themselves more to mental imagery. For the effect on choosing grouping we have no explanation.

By introducing proactive interference in a closed set of items in Experiment 2, we showed that self-reported use of mental imagery decreased. One explanation for this is that mental imagery is a form of elaboration, which is very effective for episodic LTM but not for WM (Bartsch \& Oberauer, 2021). Therefore, in a situation in which LTM suffers strong proactive interference, imagery becomes less useful, and is used less. We will go into more detail why this is not the case for sentence generation in the Interim Discussion on recall below.

Furthermore, we assessed the variability in strategy selection from trial-to-trial. Overall, subjects were more likely to switch than to stay within the same strategy. This hints at free time being used for different strategies, potentially in reaction to the material of a certain trial being easier to form a sentence, mentally imagine in a joint picture, or easier to group than to rehearse. These results suggest that reductions in adaptive strategy switching are a viable explanation for the lack of benefits in instructed strategy studies.

Finally, we observed that subjects complied to a high degree with the strategy instructions in both experiments. This observation converges with previous studies finding that young adults can implement an instructed rehearsal schedule (Souza et al., 2016), and can implement the instruction to elaborate the memoranda (Bartsch et al., 2019; 2021). These results show that the choice of a memory control process is under volitional control.

Therefore, the alternative explanation of a lack of compliance to instructions cannot explain the past lack of evidence for any of the candidate processes benefitting WM in comparison to a free baseline. 


\section{Recall}

\section{Do WM Strategies Impact LTM Recall?}

When a memory strategy is beneficial for immediate serial recall, this could be because it improves maintenance in WM, but also because it improves the accessibility of the memoranda in LTM. As we know from past literature, elaboration benefits LTM (Craik \& Tulving, 1975), whereas other strategies - like rehearsal and refreshing - have been shown to have little impact on LTM (Bartsch et al., 2018; Greene, 1987). Therefore, it is possible that elaboration improves immediate recall indirectly through creating more accessible traces in episodic LTM.

In Experiment 1 we included a delayed free-recall test to assess how WM strategy use affected the formation of LTM representations that could be accessed in a delayed free-recall test. We calculated the mean number of correctly recalled words in the delayed test per session and strategy, which can be seen in Figure 5. The Bayesian LME revealed evidence for a main effect of strategy $\left(\mathrm{BF}_{10}=2.16 \times 10^{8}\right)$, showing that reading, refreshing, and grouping were ineffective in promoting long-term recall of the words. By contrast, the two forms of elaboration, that is sentence generation and mental imagery were the only memory strategies which led to better long-term learning in Experiment 1 (see Table 3 for all BFs of the pairwise comparisons). Furthermore, there was evidence against an effect of whether the process was instructed vs. self-reported $\left(\mathrm{BF}_{01}=2606\right)$, as well as anecdotal evidence against an interaction $\left(\mathrm{BF}_{01}=1.53\right)$. 


\section{Figure 5}

Mean Number of Correctly Recalled Items in the Delayed Free Recall Test as a Function of Strategy and Session (Self-report vs. Instructed) in Experiment 1 - with an Open Pool of Items. Error bars Represent the Standard Error of the Mean.

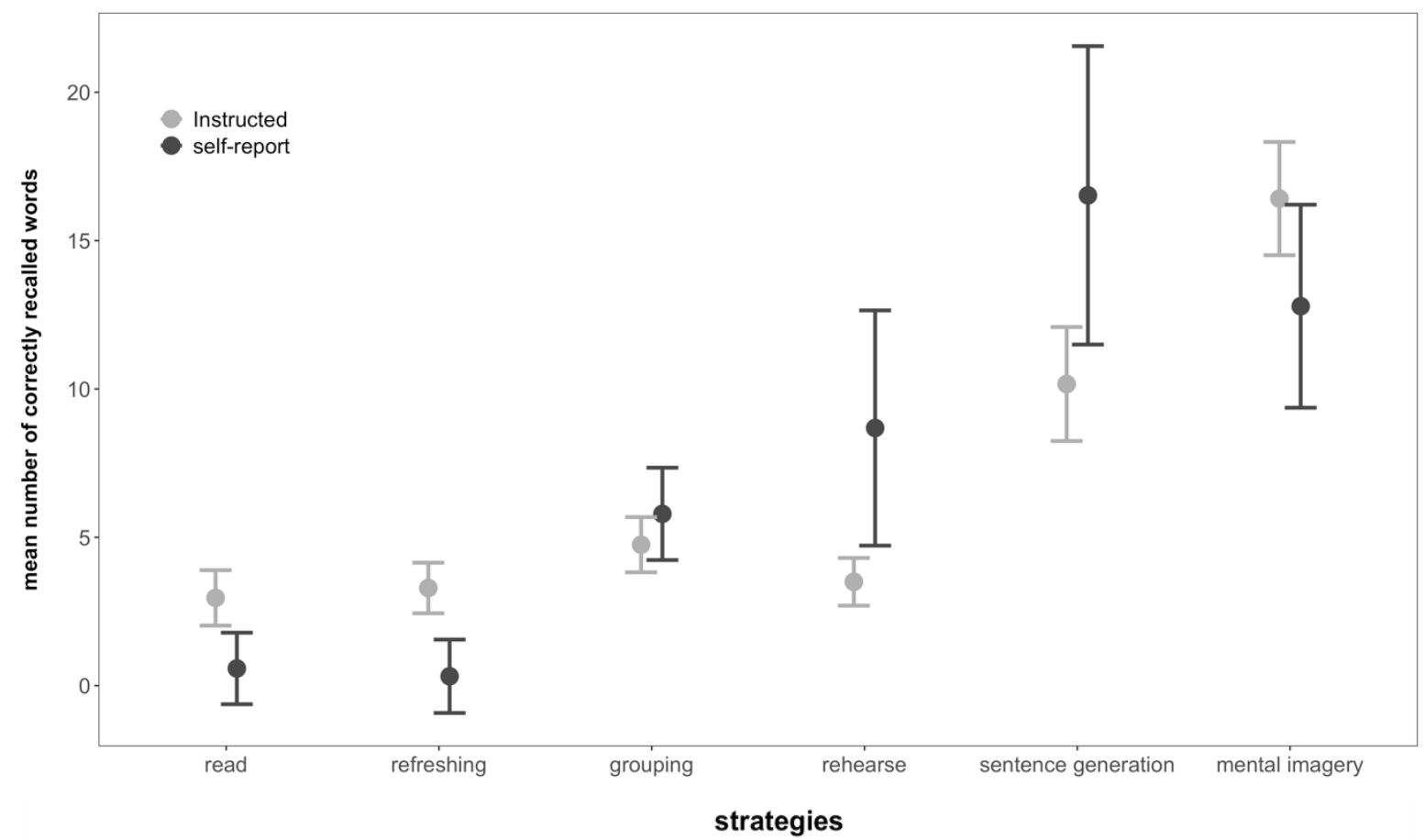

Table 3

Bayes Factors of the pairwise comparisons of strategy effects on LTM performance across sessions of Experiment 1. Bayes Factors > 3 represent substantial evidence for better performance in the strategies listed on the top compared to the ones listed on the left. Bayes Factors below 0.33 represent substantial evidence against a difference.

\begin{tabular}{|c|c|c|c|c|c|}
\hline & refreshing & rehearsal & grouping & $\begin{array}{l}\text { mental } \\
\text { imagery }\end{array}$ & $\begin{array}{l}\text { sentence } \\
\text { generation }\end{array}$ \\
\hline read & $5.97 \times 10^{-3}$ & 0.21 & 17.53 & $2.98 \times 10^{5}$ & $1.46 \times 10^{4}$ \\
\hline refreshing & & 0.44 & 10.61 & $1.96 \times 10^{5}$ & 5847 \\
\hline rehearsal & & & 0.07 & 1.94 & 2.44 \\
\hline grouping & & & & 184 & 47.37 \\
\hline mental imagery & & & & & 0.18 \\
\hline
\end{tabular}


We expected that the concreteness of the to-be-remembered words affected how beneficial some of the strategies - in particular, mental imagery -- would be for delayed memory performance. Figure 6 shows that this was the case: Concrete words benefited more from imagery, and also from sentence generation and grouping. The BGLMM provided evidence for a main effect of concreteness $\left(\mathrm{BF}_{10}=484.61\right)$, with better delayed memory performance for concrete words, as well as evidence for the interaction with Strategy $\left(\mathrm{BF}_{10}=\right.$ 6098). Follow-up analyses revealed that there were credible effects of concreteness for reading $\left(\mathrm{BF}_{10}=12.74\right)$, grouping $\left(\mathrm{BF}_{10}=13.30\right)$, sentence generation $\left(\mathrm{BF}_{10}=31.68\right)$, and mental imagery $\left(\mathrm{BF}_{10}=81.74\right)$. Instead, for rehearsal, there was evidence against an effect of concreteness $\left(\mathrm{BF}_{10}=0.30\right)$. Evidence for an effect of concreteness in case of refreshing was ambiguous $\left(\mathrm{BF}_{10}=0.82\right)$.

\section{Figure 6}

Mean Number of Correctly Recalled Items in the Delayed Free Recall Test as a Function of Strategy and Concreteness (abstract vs. concrete) in Experiment 1 - with an Open Pool of Items. Error bars Represent the Standard Error of the Mean.

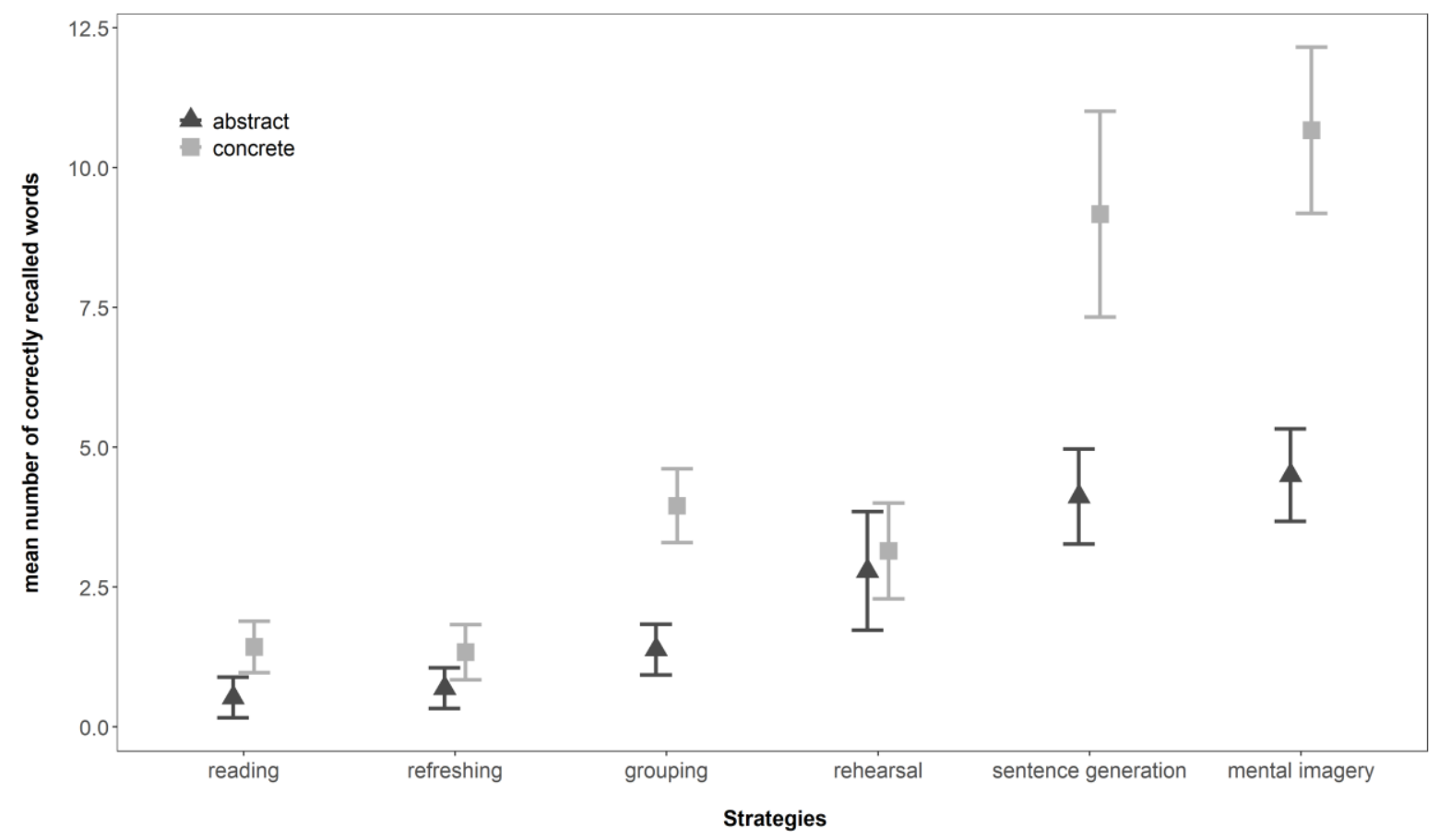




\section{Do Strategies Impact WM Performance?}

Next, we turn to the functionality of maintenance strategies for WM. We examined whether there was evidence for an overall WM cost to implementing a strategy instruction by comparing WM performance in conditions in which a strategy was self-reported vs. instructed. If there is a dual-cost of implementing instructed processes, this should lead to a cost particularly for those strategies that are cognitively demanding (i.e., refreshing and elaboration).

We looked at whether strategy choice in Session 1 has an effect on an individual's memory performance. First, to separate within-subjects from between-subjects covariation of strategy and performance, we calculated for each individual the difference between their performance on trials in which they chose a given strategy, and their mean performance (averaged over all strategies). Second, we examined the effectiveness of the different instructed strategies in Session 2 compared to each other. Third, we compared each instructed strategy (Session 2) to the mean performance in the self-report session (Session 1). This analysis replicates the comparison of instructed strategies to a "free strategy" baseline through which previous experiments have obtained evidence against a benefit of instructed strategies for WM performance.

We present analyses with mean serial recall accuracy, defined as proportion of words recalled in their correct serial position, as dependent variable. We had no a-priori hypothesis about the interaction of serial position with the effect of instructed compared to self-chosen strategies on immediate recall performance. Therefore, we have included those visualizations and analyses for the interested reader in the Online Supplementary Material.

\section{Are There Dual-Task Costs of Implementing Instructed Strategies?}

Figure 7A and B show the immediate serial recall performance over strategies and sessions in Experiment 1 and 2, respectively. The Bayesian LMEs revealed evidence for an interaction effect of session with strategy in both experiments $\left(\mathrm{E} 1: \mathrm{BF}_{10}=5.63\right.$ and $\mathrm{E} 2: \mathrm{BF}_{10}$ 
$=2.87$ ), as well as decisive evidence for both main effects in Experiment 1 (strategy: $\mathrm{BF}_{10}=$ $1.85 \times 10^{24}$ and session: $\mathrm{BF}_{10}=1.42 \times 10^{6}$ ) as well as in Experiment $2\left(\right.$ strategy: $\mathrm{BF}_{10}=5.19 \mathrm{x}$ $10^{23}$; session: $\mathrm{BF}_{10}=432$ ). In both experiments, there was overall worse performance in the instructed (E1: $M=0.61 ; S D=0.49 ; \mathrm{E} 2: M=0.64 ; S D=0.48)$ compared to the self-report session $(\mathrm{E} 1: M=0.74 ; S D=0.44 ; \mathrm{E} 2: M=0.78 ; S D=0.41)$.

In Experiment 1, the main effect of strategy was driven by better performance for grouping, mental imagery and sentence generation compared to reading and refreshing (see Table 4 for all pairwise comparisons). In Experiment 2, subjects engaged in mental imagery less frequently than in Experiment 1 (12\% vs. $25 \%$ of trials), whereas reliance on rehearsal increased (24\% to $34 \%$ ). Did this change also modulate the effectiveness of strategies for immediate serial recall? Indeed, in Experiment 2, subjects showed better performance for sentence generation compared to all other strategies, whereas mental imagery, grouping and rehearsal only led to better performance than reading and refreshing.

To answer whether there are indeed dual-tasks costs of implementing instructed strategies, we turned to the interaction effect in both Experiments separately: In Experiment 1, post-hoc comparisons of the interaction effect revealed that there was no difference between self-reported and instructed WM performance in case of rehearsal and sentence generation $\left(\mathrm{BF}_{01}=3.31\right.$ and $\mathrm{BF}_{01}=3.05$, respectively $)$. As apparent in Figure 7 by the large error bars, reading and refreshing were very rarely self-reported, leading to more uncertain performance estimation. Still, choosing these low frequency strategies resulted in better performance than when they were instructed $\left(\mathrm{BF}_{10}=35.09\right.$ and $\mathrm{BF}_{10}=15.54$, respectively). Likewise, both mental imagery and grouping resulted in better WM performance when these strategies were self-reported than instructed $\left(\mathrm{BF}_{10}=56.98\right.$ and $\mathrm{BF}_{10}=11.99$, respectively $)$. 


\section{Table 4}

Bayes Factors of the pairwise comparisons of the main effect of strategy on WM performance (across conditions) of Experiment 1 and 2. Bayes Factors $>3$ represent substantial evidence for better performance in the strategies listed in the columns compared to the strategy in the rows. $B F<1 / 3$ reflect substantial evidence against a difference between both strategies

\begin{tabular}{|c|c|c|c|c|c|c|c|c|c|c|}
\hline & \multicolumn{2}{|c|}{ refreshing } & \multicolumn{2}{|c|}{ rehearsal } & \multicolumn{2}{|c|}{ grouping } & \multicolumn{2}{|c|}{$\begin{array}{c}\text { mental } \\
\text { imagery }\end{array}$} & \multicolumn{2}{|c|}{$\begin{array}{l}\text { sentence } \\
\text { generation }\end{array}$} \\
\hline & E1 & E2 & E1 & E2 & E1 & E2 & E1 & E2 & E1 & E2 \\
\hline read & 0.14 & 0.02 & $\begin{array}{l}9.42 \\
\times 10^{6}\end{array}$ & $\begin{array}{l}2.25 \\
\times 10^{5}\end{array}$ & $\begin{array}{c}5.05 \\
\times 10^{10}\end{array}$ & $\begin{array}{l}1.42 \\
\times 10^{7}\end{array}$ & $\begin{array}{c}2.86 \\
\times 10^{11}\end{array}$ & 6010 & $\begin{array}{c}2.79 \\
\times 10^{12}\end{array}$ & $\begin{array}{c}3.91 \\
\times 10^{15}\end{array}$ \\
\hline refreshing & & & 1.35 & 7102 & 589 & $\begin{array}{l}1.88 \\
\times 10^{6}\end{array}$ & 4490 & 156 & 7736 & $\begin{array}{c}1.72 \\
\times 10^{14}\end{array}$ \\
\hline rehearsal & & & & & 12.81 & 0.01 & 78.54 & 0.026 & $\begin{array}{l}1.62 \\
\times 10^{4}\end{array}$ & 94.96 \\
\hline grouping & & & & & & & 0.12 & 0.02 & 0.02 & 31.06 \\
\hline $\begin{array}{l}\text { mental } \\
\text { imagery }\end{array}$ & & & & & & & & & 0.28 & 105 \\
\hline
\end{tabular}

In Experiment 2, post-hoc comparisons revealed that there was evidence against a recall difference between self-reported vs. instructed mental imagery and sentence generation - with the latter replicating Experiment $1\left(\mathrm{BF}_{01}=3.00\right.$ and $\mathrm{BF}_{01}=4.24$, respectively). Instruction resulted in worse performance than self-report for reading $\left(\mathrm{BF}_{10}=245\right)$ and grouping $\left(\mathrm{BF}_{10}=10.33\right)$, whereas evidence was ambiguous for rehearsal $\left(\mathrm{BF}_{10}=1.54\right)$.

Taken together, results across both Experiments 1 and 2 speak against the hypothesis that instructing strategies incurs a general dual-task cost, as there was no sign of such a cost for two of the most demanding strategies, imagery and sentence generation, whereas there was evidence for a cost for the least demanding strategy, reading. Instead, our results are better explained by our second hypothesis, namely that instructing one specific strategy (i.e., mental imagery, grouping, reading, and refreshing) impedes adaptive strategy choice and switching. This could explain why experimental studies that manipulated the engagement in 
some of these strategies across all trials have observed no evidence that they improve memory above conditions in which subjects are free to choose their own strategy.

\section{Figure 7}

Mean Immediate Serial Recall Performance Across Strategies and Session (Self-report vs. Instructed). Panel A shows the Data of Experiment 1 (Open Pool) and Panel B of Experiment 2 (Closed Pool). Error bars represent 95\% Confidence Intervals. The Order of Strategies on the x-axis Reflects the Frequency with Which They were Reported (from Least to Most Frequent).

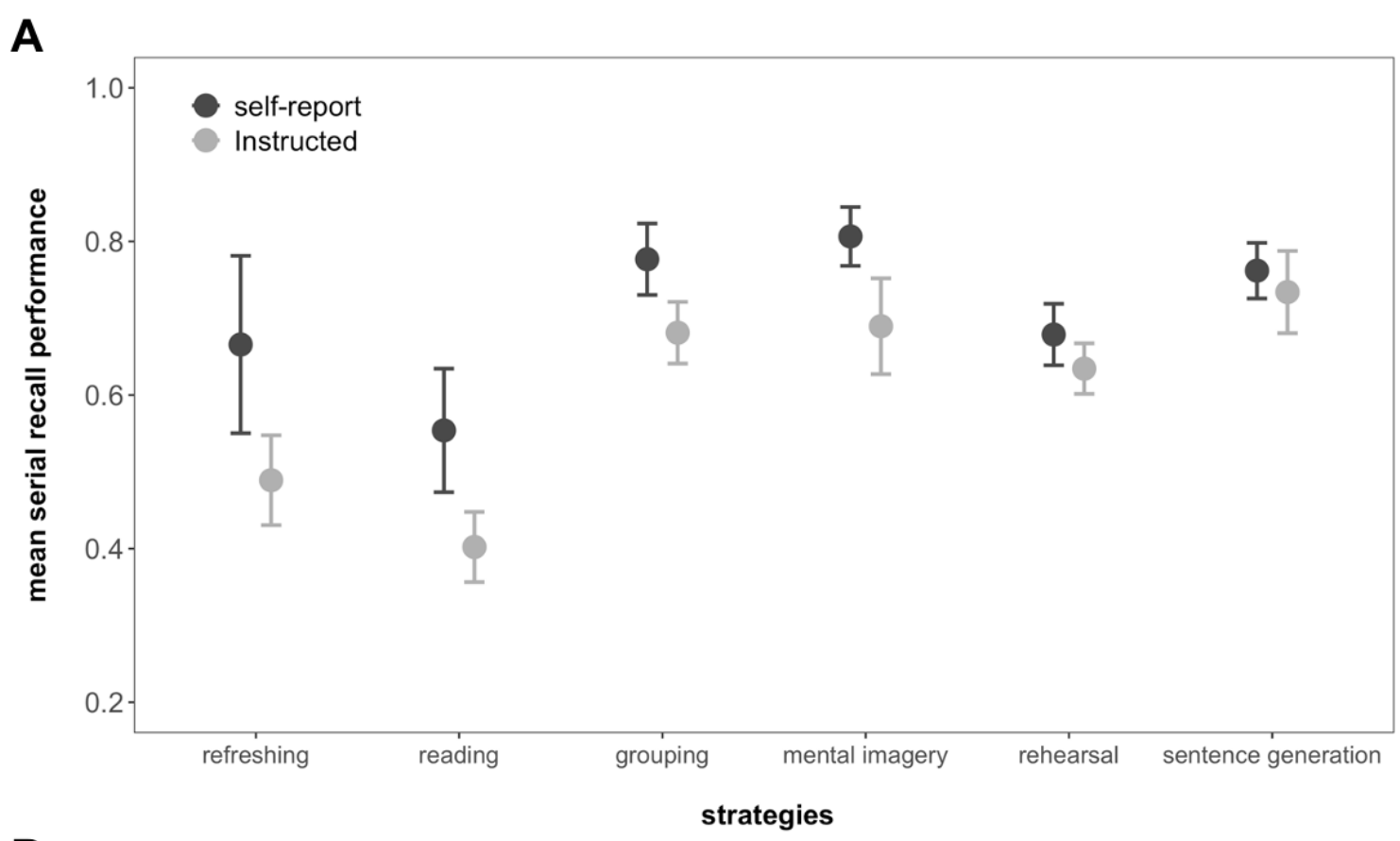

B

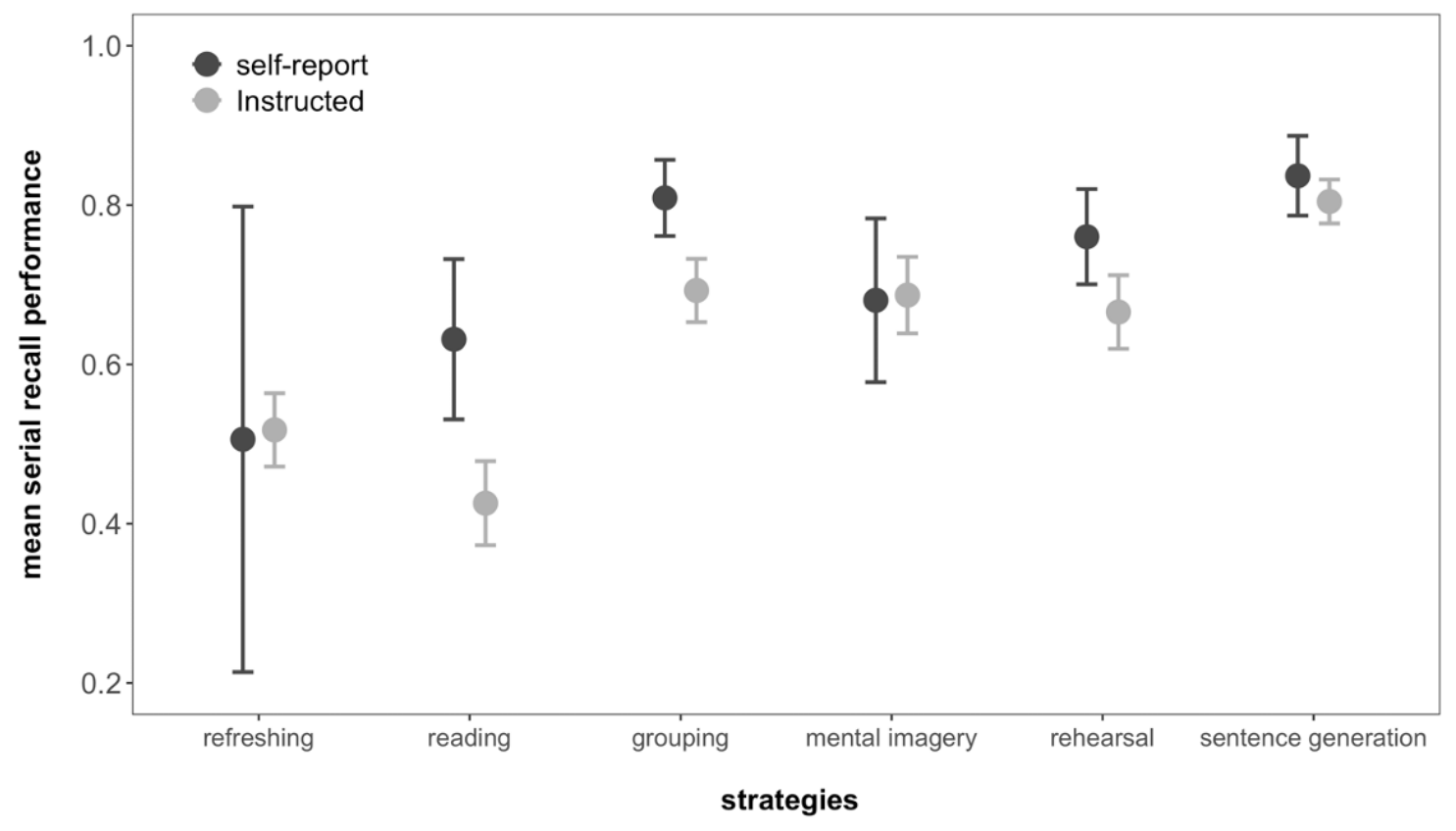




\section{Does the Choice of Strategy Make a Difference Within a Person?}

The analysis above revealed evidence for a main effect of strategy in both experiments, indicating that some of them were more beneficial to immediate serial recall performance than others. To separate within-subjects from between-subjects covariation, we calculated the effect of each strategy relative to the individuals' mean performance.

The Bayesian LMEs again revealed a main effect of strategy $\left(\mathrm{E} 1: \mathrm{BF}_{10}=4.04 \times 10^{5}\right.$ and $\left.\mathrm{E} 2: \mathrm{BF}_{10}=919\right)$. In Experiment 1 this was driven by better within-subject performance for self-reported mental imagery $\left(\mathrm{BF}_{10}=8.30\right)$ and worse performance for self-reported reading and rehearsal $\left(\mathrm{BF}_{10}=31.57\right.$ and $\mathrm{BF}_{10}=5.85$, respectively). For self-reported sentence generation, grouping, and refreshing there was ambiguous evidence for a difference compared to the individuals' mean performance $\left(\mathrm{BF}_{10}=0.63, \mathrm{BF}_{10}=0.73\right.$, and $\mathrm{BF}_{10}=0.37$, respectively).

In Experiment 2 the main effect was driven by worse performance in reading trials $\left(\mathrm{BF}_{10}=11.97\right)$. For self-reported refreshing, sentence generation, and mental imagery, evidence was inconclusive regarding differences to an individual's mean performance $\left(\mathrm{BF}_{10}=\right.$ $0.88, \mathrm{BF}_{10}=1.15$, and $\mathrm{BF}_{10}=0.81$, respectively). For rehearsal and grouping, there was some evidence against a difference to an individual's mean performance $\left(\mathrm{BF}_{01}=3.33\right.$, and $\mathrm{BF}_{01}=$ 3.22).

Taken together, the results of Experiment 1 hint at mental imagery being beneficial to WM performance compared to the other memory control processes - even when considering the individuals' mean performance. Processes like reading and rehearsal instead lead to worse performance within individuals. Therefore, based on the findings using an open item pool there seems to be some self-chosen strategies that yield benefits whereas others produce costs within a person. Yet when handling a closed pool of items in Experiment 2, making it hard to rely on episodic LTM traces, the benefit of mental imagery vanished. Here, most strategies 
were associated with comparable performance, with reading as the only exception, which continued to produce a cost.

In summary, the results of both experiments together don't hint at any strategy being beneficial to WM performance compared to an individuals' mean performance. Reading instead leads to worse performance within individuals. In a situation that affords little reliance on episodic LTM, there appears to be no strategy that participants can make use of to boost their memory relative to what they can accomplish with another strategy.

\section{Which Instructed Strategy is More Beneficial?}

Next, we were interested in the effect of instructed strategies on WM recall. There was decisive evidence for a main effect of instructed strategy in both experiments $\left(\mathrm{E} 1: \mathrm{BF}_{10}=3.16\right.$ $\mathrm{x} 10^{24}$ and $\mathrm{E} 2: \mathrm{BF}_{10}=1.42 \times 10^{22}$ ). Table 5 presents BFs of the follow-up pairwise comparisons of the instructed strategies in Session 2. These effects revealed that in both experiments instructing reading led to worse performance than all other strategies, and instructed refreshing led to worse performance than rehearsal, grouping, mental imagery, and sentence generation. Instructed rehearsal, grouping, and mental imagery led to similar performance. The change from on open to a closed pool of items from Experiment 1 to 2 changed the effect of instructed strategies with regard to mental imagery, which in a closed pool of items yielded worse performance than sentence generation - whereas with an open pool it resulted in similar performance. 


\section{Table 5}

Bayes Factors of the pairwise comparisons of instructed-strategy effects on WM performance in Session 2 of Experiment 1 and 2. Bayes Factors > 3 represent substantial evidence for better performance in the strategies listed on the top compared to the ones listed on the left.

\begin{tabular}{|c|c|c|c|c|c|c|c|c|c|c|}
\hline & \multicolumn{2}{|c|}{ refreshing } & \multicolumn{2}{|c|}{ rehearsal } & \multicolumn{2}{|c|}{ grouping } & \multicolumn{2}{|c|}{$\begin{array}{c}\text { mental } \\
\text { imagery }\end{array}$} & \multicolumn{2}{|c|}{$\begin{array}{l}\text { sentence } \\
\text { generation }\end{array}$} \\
\hline & E1 & E2 & E1 & E2 & E1 & E2 & E1 & E2 & E1 & E2 \\
\hline read & 2.48 & 4.62 & $\begin{array}{l}5.44 \\
\times 10^{7}\end{array}$ & $\begin{array}{l}2.52 \\
\times 10^{7}\end{array}$ & $\begin{array}{c}1.63 \times \\
10^{8}\end{array}$ & $\begin{array}{l}2.15 \\
\times 10^{8}\end{array}$ & $\begin{array}{c}7.26 \times \\
10^{6}\end{array}$ & $\begin{array}{c}7.85 \times \\
10^{8}\end{array}$ & $\begin{array}{c}1.46 \times \\
10^{8}\end{array}$ & $\begin{array}{c}5.99 \times \\
10^{15}\end{array}$ \\
\hline refreshing & & & 218 & 742 & $\begin{array}{c}1.14 \times \\
10^{4}\end{array}$ & $\begin{array}{l}1.31 \\
\times 10^{4}\end{array}$ & 2719 & 2542 & $\begin{array}{c}8.96 \times \\
10^{4}\end{array}$ & $\begin{array}{c}1.43 \times \\
10^{10}\end{array}$ \\
\hline rehearsal & & & & & 0.60 & 0.24 & 0.60 & 0.31 & 6.99 & 1317 \\
\hline grouping & & & & & & & 0.35 & 0.15 & 0.54 & 2333 \\
\hline $\begin{array}{l}\text { mental } \\
\text { imagery }\end{array}$ & & & & & & & & & 0.54 & 4718 \\
\hline
\end{tabular}

To compare these effects of instructed strategies to a "free baseline", as done in previous studies (Bartsch et al., 2018; Bartsch \& Oberauer, 2021; Souza \& Oberauer, 2018, 2020), we next compared performance with each instructed strategy to the mean serial-recall performance from the self-report sessions (Session 1) in each experiment. None of the instructed strategies surpassed the mean serial-recall performance of the session with free strategy choice, consistent with previous literature. In Experiment 1, instructed reading $\left(\mathrm{BF}_{10}\right.$ $\left.=2.94 \times 10^{7}\right)$, refreshing $\left(\mathrm{BF}_{10}=2.21 \times 10^{4}\right)$, and rehearsal $\left(\mathrm{BF}_{10}=133\right)$ led to worse performance than in Session 1, and instructed sentence generation yielded equivalent performance to the mean performance in Session $1\left(\mathrm{BF}_{01}=4.65\right)$. There was anecdotal evidence against a difference in case of instructed mental imagery and grouping $\left(\mathrm{BF}_{01}=2.35\right.$, $\mathrm{BF}_{01}=1.42$ respectively). In Experiment 2, instructed reading $\left(\mathrm{BF}_{10}=2.42 \times 10^{9}\right)$, refreshing $\left(\mathrm{BF}_{10}=4.09 \times 10^{6}\right)$, rehearsal $\left(\mathrm{BF}_{10}=428\right)$, mental imagery $\left(\mathrm{BF}_{10}=106\right)$, and grouping $\left(\mathrm{BF}_{10}\right.$ $=33.79$ ) produced worse performance compared to Session 1 , whereas instructed sentence 
generation yielded anecdotal evidence for equivalent performance to the mean performance in Session $1\left(\mathrm{BF}_{01}=2.71\right)$.

\section{Does Concreteness Interact with the Effectiveness of Strategies?}

One could expect that the concreteness of the to-be-remembered words affected how beneficial a certain strategy would be for immediate memory performance. We have seen above that people in Experiment 1 adapted their choice of strategy based on the word concreteness. Do these strategies aid more or less in recalling concrete vs. abstract words? And critically, is there evidence for an interaction, in the direction that the concreteness differentially affects some strategies but not others? To investigate this, we entered concreteness as a factor to the BGLMM. Although there was clear evidence for a main effect of concreteness $\left(\mathrm{BF}_{10}=1.8 \times 10^{13}\right.$, see also Figure 8), concreteness did not credibly enter any of the interactions (Concreteness x Strategy: $\mathrm{BF}_{10}=0.06$, Concreteness $\mathrm{x}$ Session: $\mathrm{BF}_{10}=$ 0.14, Three-way interaction: $\left.\mathrm{BF}_{10}=0.07\right)$. These results indicate that abstract words are in general more difficult to recall correctly in an immediate memory test, yet that this holds independent of which strategy was employed on the memoranda, and independently of whether these strategies were self-chosen or instructed. 


\section{Figure 8}

Mean Immediate Serial Recall Performance Across Strategies and Concreteness (Abstract vs. Concrete). Error bars represent 95\% Confidence Intervals. The Order of Strategies on the x-axis Reflects the Frequency with Which They were Reported (from Least to Most Frequent).

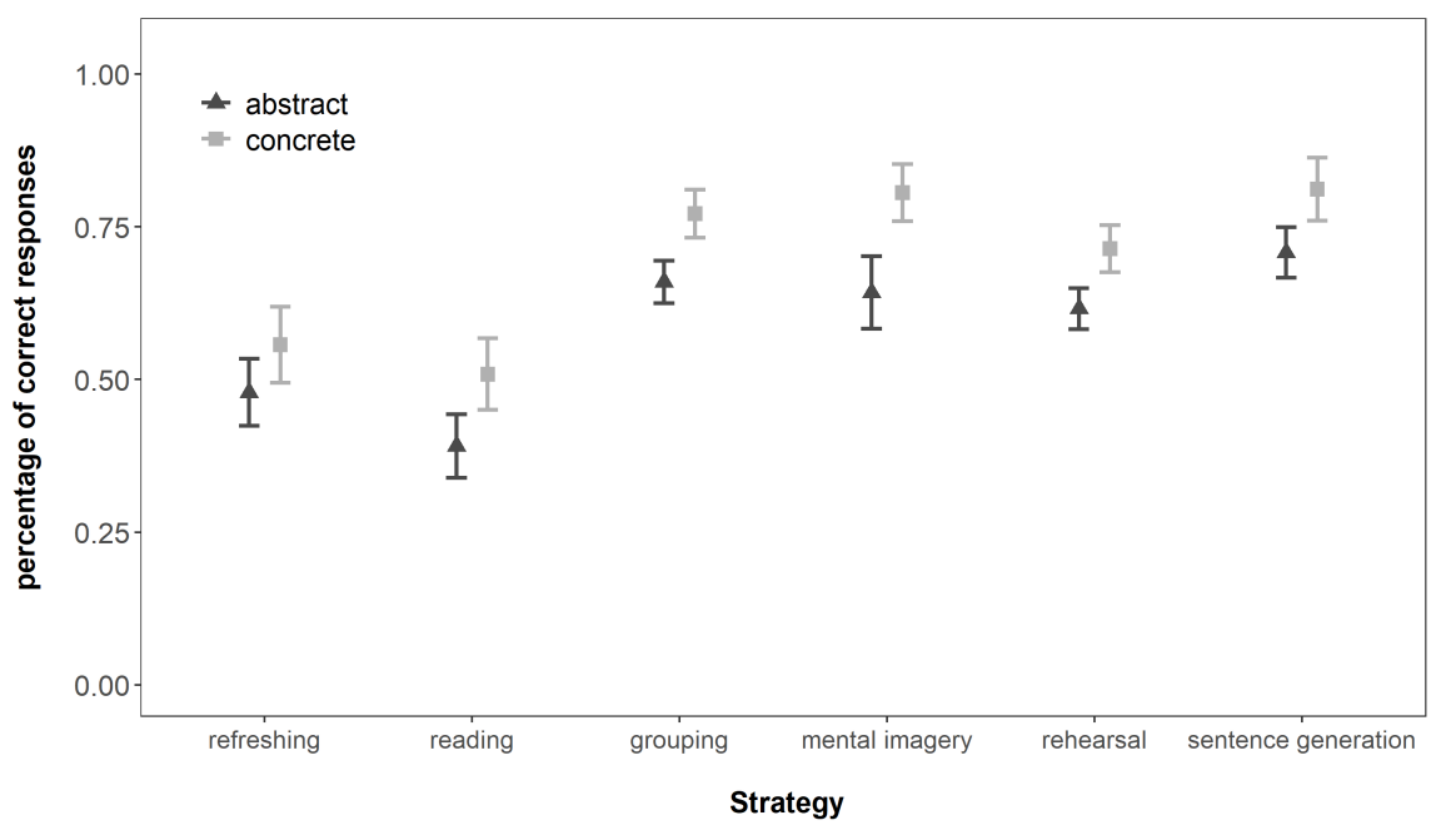

Interim Discussion: Recall as a Function of Strategy Use

Previous studies assessed the causal effect of instructing maintenance strategies compared to a free baseline in which subjects were free to use whatever strategy they preferred, and observed no beneficial effects of instructing a strategy (Bartsch \& Oberauer, 2021; Souza \& Oberauer, 2018, 2020). Here, we investigated whether this could be explained by (1) secondary task demands that the implementation of a strategy instruction potentially entails, or (2) instructions preventing participants from adaptively choosing different strategies from trial to trial.

We examined this question by comparing serial recall performance in trials in which a WM strategy was self-reported vs. instructed. We observed that for some strategies (i.e., reading, refreshing, mental imagery [only in E1], and grouping), there was a detrimental effect of instruction implementation. For the most frequently reported strategies, namely sentence generation and rehearsal, performance was similar between self-reported and instructed conditions. 
Taken together, these results do not support the hypothesis that instructed strategies impose a dual-task cost. Such a dual-task cost would either be equal for all strategies - if it simply reflected the cost of maintaining and implementing any instruction - or it would be highest for the cognitively most demanding strategies, namely refreshing, imagery, and sentence generation, and negligible for undemanding strategies such as reading. The observed pattern of costs across both Experiments 1 and 2 does not match either of these predictions.

Instead, the results are better explained by instructions preventing participants from adaptively choosing different strategies from trial to trial. Sentence generation and rehearsal could be applied more successfully than other strategies to a relatively large number of trials as indicated by their higher frequency in self-reports. Therefore, instructing participants to use these strategies consistently does not force them to use an unsuitable strategy on many trials, and therefore does not lead to a performance decrement. The other strategies are not helpful on most of the trials in which participants were instructed to use them. Therefore, using them only on suitable lists is more beneficial than using them consistently on all lists, as demanded in the instruction condition.

In order to replicate the pattern of results of previous instruction studies we compared the performance of each instructed strategy to the individuals' mean performance across all self-report trials - mimicking the free baseline condition of previous instruction studies. That analysis revealed that also in the present study none of the instructed strategies surpassed the performance in a free baseline condition. This observation agrees well with the assumption that in the free baseline condition participants choose their strategies adaptively. On some trials, they choose strategies associated with poor performance, such as reading, or refreshing. Yet, doing so does not appear to be detrimental to overall memory performance compared to an instructed-strategy condition in which they use one of the better strategies (elaboration, grouping) throughout. This can be explained by assuming that the better strategies are helpful only on a subset of trials, and these are the trials on which participants choose those strategies. 
On other trials, these strategies yield no benefit, and therefore instructing participants to use elaboration or grouping on all trials does not lift overall performance above the free baseline.

In Experiment 1 we found that instructed mental imagery and sentence generation yielded benefits for immediate serial recall performance compared to instructed reading, refreshing and, partly, rehearsal. Yet, as these two processes were also the only ones which led to better LTM, we wanted to investigate in Experiment 2 whether they directly benefited WM maintenance, or indirectly assisted immediate recall by improving episodic LTM. In Experiment 2, we decreased the usefulness of relying on LTM representations by reducing the pool of stimuli to a closed set of only 12 words that were repeated over the course of the experiment, thereby inducing proactive interference.

In Experiment 2, self-reports of mental imagery were reduced, and performance in these trials was worse than for sentence generation, and equivalent to instructed rehearsal and grouping. This suggests that the beneficial effect of mental imagery in Experiment 1 could be explained by the formation of stronger episodic LTM traces, which improved both immediate as well as delayed recall performance. With unique memoranda in every trial, mental imagery has a clear advantage because distinct LTM representations of the self-created images can be created for every episode, benefiting item memory. As unique episodes cannot be created with a closed pool of repeating items, subjects engage in this strategy less frequently.

Yet why did sentence generation remain indifferent to inducing proactive interference? One explanation is that sentences - in contrast to mental images - preserve the order of the individual items, which is especially crucial in case the same items are repeated over the course of different trials (yet at varying serial positions). Forming a mental image does not preserve this order.

Further, by forming sentences of the memoranda, the level of proactive interference between trials can actually be reduced. Specifically, including a word in a specific role in a new sentence can increase the uniqueness of this episode, distinguishing this instance of the 
word from its many previous occurrences. For example, for remembering the words Pony Tooth-Coffee - Ball, one could form the sentence: The pony loses its tooth in the coffee after being hit by a white ball. In the next trial, the memoranda could be Coffee - Tooth - Ball Pony. Now, one can form the sentence: Drinking my hot coffee hurts my tooth, but later I can play ball more energetically with my pony. From the first example sentence to the second, the context of each of the words has changed: For instance, the coffee is either made undrinkable by a tooth or the person is drinking it in the morning. Therefore, sentence generation seems to facilitate both item memory and order memory, even under conditions of proactive interference, which makes it still effective for immediate serial recall.

To conclude, the results of Experiment 2 suggest that different forms of elaboration are differently susceptible to manipulations of proactive interference: Mental imagery becomes less effective, but sentence generation remains effective. The fact that proactive interference between trials modulates the effectiveness of elaboration strategies for immediate serial recall confirms our assumption that elaboration is helpful for performance on a WM test indirectly through boosting episodic LTM, which contributes to immediate recall.

\section{General Discussion}

Based on theoretical assumptions about a causal role of memory control processes for maintenance in WM, as well as correlational evidence on the effectiveness of maintenance strategies on WM performance, previous experimental research has attempted to provide causal evidence that these processes benefit memory. These approaches were based on the hypotheses that instructing a specific strategy would induce any individual to engage in successful strategies more, or to switch from a less effortful strategy (e.g., reading) to one that requires more processing, but which leads to a larger mnemonic benefit (e.g., sentence generation). Yet, both approaches failed to provide evidence in favor of a beneficial effect of any of these strategies. 
Our main aim for the present work was to examine reasons for the discrepancies between, on the one hand, correlational studies pointing to benefits of some maintenance strategies for WM performance and, on the other hand, experimental studies indicating no causal effect of these strategies.

\section{Dual-Task Costs Cannot Explain Lack of Benefits}

Previous experimental studies have compared performance under instructed strategy conditions to a no-instruction baseline, generally failing to find performance differences between them (Bartsch et al., 2018; Souza \& Oberauer, 2018, 2020). One possible explanation for this outcome is that strategy instructions create dual-task costs. So far, no previous studies directly compared self-reported to instructed strategy use to test this possibility.

Across two experiments, comparisons of an instructed strategy condition to a free baseline including trial-to-trial strategy reports revealed no costs of instructing the most commonly reported maintenance strategies, namely rehearsal and sentence generation. Other reported strategies such as reading, refreshing, mental imagery, and grouping showed instruction implementation-costs. In order to uphold the dual-task-cost explanation for the finding that experimental investigations using instructions never reached the performance of a free baseline, one would have to assume that mental imagery, reading and refreshing impose a dual-task cost, whereas sentence generation and rehearsal do not. Whereas this could plausibly be true for refreshing compared to rehearsal (see e.g. Vergauwe, Camos, \& Barrouillet, 2014), it is very implausible for the contrast between sentence generation and mental imagery. It is also very implausible that implementing the instruction to merely read each word as it is presented imposes a dual-task cost because that instruction is the only one that does not even add a secondary task: Participants have to read the words to do the memory task anyway. Therefore, the finding that experimental investigations using instructions not 
showing a benefit for WM performance can't be plausibly explained by the instruction imposing a secondary task cost.

\section{Adaptive Strategy Switching is Beneficial for Memory}

The second possibility we addressed in the present study is that instructed strategies failed to benefit memory performance because they prevent adaptive switching between strategies. The recall performance, as well as the high frequency of switches between strategies reported in Session 1 of Experiments 1 and 2, indicate that in a free-baseline, participants naturally choose their strategy adaptively on a trial-by-trial basis. According to this explanation, for each strategy, the trials on which they self-reported a strategy are the ones for which this strategy is relatively successful - more successful than when the same strategy is applied to all trials. The difference to instructed trials becomes most apparent for the strategies that are rarely chosen, because in those cases, the majority of trials in the instructed-strategy condition are not particularly suitable for that strategy.

What drives the choice of strategies from trial to trial? By definition, a strategy is a process under the person's volitional control, and participants' ability to comply with our strategy instructions testifies to that. On what basis do they make their choices? A first question to ask in this context is whether they decide upon the strategy for the next trial already before presentation of the memory list (a form of proactive control; Braver, 2012), or only later, after having seen at least the first few items? In the latter case, is plausible that the strategy is selected in part to be suited for the content of the memory list. For instance, some words, or sets of words, are easier to elaborate than others. This assumption motivated our variation of concreteness of list words in Experiment 1. Indeed, participants chose mental imagery and grouping more often on lists with concrete words than lists with abstract words (Supplementary Figure S9). Despite these differences in choice behavior, the effect of concreteness did not interact with the type of strategy chosen when it came to immediate memory performance. This fits with our observation that strategy switches also did not seem 
to be motivated by their recall performance in the preceding trial (see Online Supplementary Materials for details). Future experiments could investigate the effects of other aspects of list items, or list composition, on strategy choice by varying these features of the memoranda systematically between trials.

As we kept the order of sessions constant to avoid any influence of the strategy instructions on the pattern of self-chosen strategies by the participants, one might wonder whether our results on the direct comparison between self-reported and instructed strategy use are compromised by this design choice. We argue that our findings cannot be explained by the constant session order (self-report first, instructed strategies second). Given that the two sessions were completed on different days, we can rule out fatigue effects across sessions. To rule out fatigue effects within a session - especially as the second session included more trials than the first - we analyzed performance as a function of trial, which yielded evidence against an interaction effect of session with trial number $\left(\right.$ Exp.1: $\mathrm{BF}_{01}=4.24$ and Exp. $2: \mathrm{BF}_{01}=$ 649.65; see Online Supplementary Materials for details). Finally, the only remaining plausible effect of session order is a practice effect, which would have led to an advantage for performance in the instructed strategy condition in Session 2. In contrast, our results go in the opposite direction, showing that performance with instructed strategies (Session 2) was worse than when participants were to freely chose the strategy to use (Session 1).

Previous experiments instructing individual control processes show that using one strategy for all subjects and trials does not yield an overall benefit. The results across both experiments here revealed that choosing the best strategy for each trial by each person leads to a benefit, relative to the choice of a single strategy by everyone on every trial. Subjects seem to flexibility switch strategies from trial to trial. One interpretation would be that subjects choose an optimal process based on the material and their own capacity. Strategy choice depending on one's own capacity is supported by correlational studies in which subjects engaging in strategies like mental imagery or sentence generation showed higher 
simple span as well as complex span performance - which could reflect the phenomenon that subjects with larger WM capacity can afford to engage in more effortful processes (e.g., Bailey et al., 2008, 2011).

In conclusion, dual-task costs are unlikely to explain the lack of improvements for the mostly common assessed strategies. The ineffectiveness of strategy instructions seems more related to them impeding adaptive strategy choices.

\section{Do Strategies Explain Why Free Time Benefits Serial Recall?}

Presenting memory items at a slower rate - thus adding free time between them leads to better performance in simple span (Tan \& Ward, 2008; Oberauer, in press) and complex span tasks (Barrouillet et al., 2011), and some researchers have explained this effect by assuming that free time is used for memory control processes assisting maintenance (Barrouillet et al., 2011; Tan \& Ward, 2008). The present experiments provided three new insights into why WM benefits from longer free time between items: (1) we learned what participants indicate to be doing during this time, and (2) how this varies from trial to trial, and (3) we have reason to believe that part of the benefit is probably driven by LTM.

First, subjects report using free time for elaborative strategies such as mental imagery and sentence generation on around $62 \%$ of the trials. This percentage was much higher than reported in previous studies, in which mental imagery was reported for about 6-14\% of the trials, and sentence generation 2-13\% (see Bailey, Dunlosky, \& Hertzog, 2009; Bailey et al., 2008, 2011 for details). One reason for this shift could lie in the longer free-time interval implemented here: Within 4.5 seconds of free time between items, subjects have much more time to use time-demanding strategies than in most studies of immediate serial recall. Further, rehearsal was among the most commonly reported strategies (24-34\% of trials), yet resulting in no benefit on performance in the WM task within an individual. These results match previous findings of self-report studies having found that that rehearsal is an ineffective 
maintenance strategy (Bailey et al., 2011; Dunlosky \& Kane, 2007), and also converge with experimental evidence for the ineffectiveness of rehearsal (Souza \& Oberauer, 2018, 2020). Taken together, participants indicate to engage in rehearsal during free time, yet again we present evidence that this does not boost WM performance. In summary, our first insight was that one reason why free time between items is beneficial to WM performance is that participants use this time to engage more in effective strategies (i.e., elaboration), and less in ineffective ones (i.e., rehearsal). This is the case specifically in situations under which elaboration is indeed effective - meaning when remembering word lists with an open pool.

Second, we have shown that in WM tasks with generous free time, a mixture of memory strategies is used, and that subjects switch between them from trial to trial. If there is a benefit of strategies on WM performance, it seems not to be driven by one single strategy applied consistently. Some strategies are more beneficial when participants can spontaneously select them, as it was the case of grouping and mental imagery. We further showed that although people were more likely to switch than to stay with the same memory strategy from trial-to-trial, they did show a higher tendency to stick with strategies that were more beneficial to performance than to switch to any specific other strategy. Potentially, subjects are able to react to the material and choose optimal encoding strategies when given enough time. This is not the case for all strategies though, as rehearsal and sentence generation generated similar performance levels irrespective of being self-reported or instructed, even though in the self-report condition there was more variability in strategy use over trials. In sum, the second reason why free time is beneficial is because it provides time to adapt strategies to the memoranda presented.

Third, we showed that part of the benefit of maintenance strategies for performance on WM tasks is probably driven by LTM. In Experiment 1, self-reported mental imagery was found to boost immediate serial recall performance. Yet, this was no longer the case in Experiment 2 in which a closed pool of items was repeatedly used, thereby reducing the 
usefulness of item memory, and increasing demands on serial order memory. This indicates that mental imagery helps immediate recall through the formation of LTM traces of the items. The same is probably true for other elaborative strategies that are known to improve episodic LTM, such as sentence generation, although the present Experiment 2 was not suited to show that, because sentence generation helps to mitigate proactive interference.

\section{Conclusion}

Across two experiments we have shown that instructing maintenance strategies that are correlated with poor performance in self-reported sessions leads to worse immediate serial recall than when participants freely choose their strategies. By contrast, instructing strategies that were correlated with good performance (e.g., Exp 1: mental imagery $[M=68.96 \%$ correct $]$ and sentence generation $[M=73.41 \%$ correct $]$ ) did not lead to better performance than participant's freely chosen strategy-mix $(M=74.20 \%$ correct $)$. Without strategy instructions, participants frequently switch between strategies, and apparently do so adaptively. Among the strategies leading to the best performance are variants of elaboration. The benefit of these strategies for performance on a WM test is probably driven by their effect on episodic LTM. Our results show that we cannot push participants to do better than what they already manage to do on their own: instructing "effective strategies" can, at best, yield the same level of performance as when they are free to choose their own strategy. 


\section{References}

Atkinson, R. C., \& Shiffrin, R. M. (1968). Human memory: A proposed system and its control processes. In Psychology of learning and motivation (Vol. 2, Issue C, pp. 89195). Elsevier. https://doi.org/10.1016/S0079-7421(08)60422-3

Baddeley, A. D. (1986). Working Memory. In Current Biology (Vol. 20, Issue 4). Oxford University Press. https://doi.org/10.1016/j.cub.2009.12.014

Bailey, H., Dunlosky, J., \& Hertzog, C. (2009). Does differential strategy use account for agerelated deficits in working-memory performance? Psychology and Aging, 24(1), 82-92. https://doi.org/10.1037/a0014078

Bailey, H., Dunlosky, J., \& Kane, M. J. (2008). Why does working memory span predict complex cognition? Testing the strategy affordance hypothesis. Memory \& Cognition, 36(8), 1383-1390. https://doi.org/10.3758/MC.36.8.1383

Bailey, H., Dunlosky, J., \& Kane, M. J. (2011). Contribution of strategy use to performance on complex and simple span tasks. Memory \& Cognition, 39(3), 447-461. https://doi.org/10.3758/s13421-010-0034-3

Barr, D. J., Levy, R., Scheepers, C., \& Tily, H. J. (2013). Random effects structure for confirmatory hypothesis testing: Keep it maximal. Journal of Memory and Language, 68(3), 255-278. https://doi.org/10.1016/j.jml.2012.11.001

Barrouillet, P., Gorin, S., \& Camos, V. (2020). Simple Spans Underestimate Verbal Working Memory Capacity. Journal of Experimental Psychology: General. https://doi.org/10.1037/xge0000957

Bartsch, L. M., Loaiza, V. M. V. M., Jäncke, L., Oberauer, K., \& Lewis-Peacock, J. A. J. A. (2019). Dissociating refreshing and elaboration and their impacts on memory. NeuroImage, 199, 585-597. https://doi.org/10.1016/j.neuroimage.2019.06.028

Bartsch, L. M., \& Oberauer, K. (2021). The effects of elaboration on working memory and 
long-term memory across age. Journal of Memory and Language, 118, 104215. https://doi.org/10.1016/j.jml.2020.104215

Bartsch, L. M., Singmann, H., \& Oberauer, K. (2018). The effects of refreshing and elaboration on working memory performance, and their contributions to long-term memory formation. Memory \& Cognition, 46(5). https://doi.org/10.3758/s13421-0180805-9

Braver, T. S. (2012). The variable nature of cognitive control: a dual mechanisms framework. Trends in Cognitive Sciences, 16(2), 106-113. https://doi.org/https://doi.org/10.1016/j.tics.2011.12.010

Broadbent, D. E., \& Broadbent, M. H. P. (1981). Articulatory suppression and the grouping of successive stimuli. Psychological Research, 43(1), 57-67.

Camos, V., Johnson, M. R., Loaiza, V. M., Portrat, S., Souza, A. S., \& Vergauwe, E. (2018). What is attentional refreshing in working memory? Annals of the New York Academy of Sciences, 1-14. https://doi.org/10.1111/nyas.13616

Camos, V., \& Portrat, S. (2015). The impact of cognitive load on delayed recall. Psychonomic Bulletin \& Review, 22(4), 1029-1034. https://doi.org/10.3758/s13423-014-0772-5

Craik, F. I. M., \& Tulving, E. (1975). Depth of Processing and the Retention of Words in Eisodic Memory. Journal of Experimental Psychology: General, 104(3), 268-294.

Dunlosky, J., \& Kane, M. J. (2007). The contributions of strategy use to working memory span: A comparison of strategy assessment methods. The Quarterly Journal of Experimental Psychology, 60(9), 1227-1245.

https://doi.org/10.1080/17470210600926075

Farrell, S. (2008). Multiple roles for time in short-term memory: Evidence from serial recall of order and timing. Journal of Experimental Psychology: Learning, Memory, and Cognition, 34(1), 128.

Greene, R. L. (1987). Effects of maintenance rehearsal on human memory. Psychological 
Bulletin, 102(3), 403-413. https://doi.org/10.1037/0033-2909.102.3.403

Hartley, T., Hurlstone, M. J., \& Hitch, G. J. (2016). Effects of rhythm on memory for spoken sequences: A model and tests of its stimulus-driven mechanism. Cognitive Psychology, $87,135-178$.

Hitch, G. J., Burgess, N., Towse, J. N., \& Culpin, V. (1996). Temporal Grouping Effects in Immediate Recall: A Working Memory Analysis. THE QUARTERLY JOURNAL OF EXPERIMENTAL PSYCHOLOGY, 49(1), 116-139.

Johnson, M. K., Reeder, J. a, Raye, C. L., \& Mitchell, K. J. (2002). Second thoughts versus second looks: an age-related deficit in reflectively refreshing just-activated information. Psychological Science : A Journal of the American Psychological Society / APS, 13(1), 64-67. https://doi.org/10.1111/1467-9280.00411

Jonker, T. R., \& Macleod, C. M. (2015). Disruption of relational processing underlies poor memory for order. Journal of Experimental Psychology : Learning, Memory, and Cognition, 41(3), 831-840. https://doi.org/http://dx.doi.org/10.1037/xlm0000069

Kass, R. E., \& Raftery, A. E. (1995). Bayes factors. Journal of the American Statistical Association, 90(430), 773-795. https://doi.org/10.1080/01621459.1995.10476572

Liu, Y. S., \& Caplan, J. B. (2020). Temporal grouping and direction of serial recall. Memory \& Cognition, 48(7), 1295-1315.

Loaiza, V. M., \& Lavilla, E. T. (2021). Elaborative strategies contribute to the long-term benefits of time in working memory. Journal of Memory and Language, 117(December 2020), 104205. https://doi.org/10.1016/j.jml.2020.104205

Loaiza, V. M., \& Mccabe, D. P. (2012). Temporal-contextual processing in working memory: Evidence from delayed cued recall and delayed free recall tests. Memory \& Cognition, 40(2), 191-203. https://doi.org/10.3758/s13421-011-0148-2

McNamara, D. S., \& Scott, J. L. (2001). Working memory capacity and strategy use. Memory and Cognition, 29(1), 10-17. https://doi.org/10.3758/BF03195736 
Morey, R. D., Rouder, J. N., \& Jamil, T. (2015). BayesFactor: Computation of Bayes factors for common designs. $R$ Package Version 0.9, 9.

Morrison, A. B., Rosenbaum, G. M., Fair, D., \& Chein, J. M. (2016). Variation in strategy use across measures of verbal working memory. Memory \& Cognition, 44(6), 922-936. https://doi.org/10.3758/s13421-016-0608-9

Naveh-Benjamin, M., \& Jonides, J. (1984). Maintenance rehearsal: A two-component analysis. Journal of Experimental Psychology: Learning, Memory, and Cognition, 10(3), 369.

Oberauer, K. (2019). Is Rehearsal an Effective Maintenance Strategy for Working Memory? Trends in Cognitive Sciences, 23(9), 798-809. https://doi.org/10.1016/j.tics.2019.06.002

R Core Team. (2017). R: A language and environment for statistical computing. Vienna, Austria; 2014. https://www.r-project.org/.

Richardson, J. T. E. (1998). The availability and effectiveness of reported mediators in associative learning: A historical review and an experimental investigation. Psychonomic Bulletin \& Review, 5(4), 597-614.

Rouder, J. N. (2014). Optional stopping: No problem for Bayesians. Psychonomic Bulletin \& Review, 21(2), 301-308.

Ryan, J. (1969a). Grouping and short-term memory: different means and patterns of grouping. The Quarterly Journal of Experimental Psychology, 21(2), 137-147. https://doi.org/10.1080/14640746908400206

Ryan, J. (1969b). Temporal grouping, rehearsal and short-term memory. The Quarterly Journal of Experimental Psychology, 21(2), 148-155.

Souza, A. S., \& Oberauer, K. (2017). The contributions of visual and central attention to visual working memory. Attention, Perception, \& Psychophysics. https://doi.org/10.3758/s13414-017-1357-y

Souza, A. S., \& Oberauer, K. (2018). Does articulatory rehearsal help immediate serial recall? 
Cognitive Psychology. https://doi.org/10.1016/j.cogpsych.2018.09.002

Souza, A. S., \& Oberauer, K. (2020). No Evidence That Articulatory Rehearsal Improves Complex Span Performance. Journal of Cognition, 3(1), 1-25. https://doi.org/10.5334/joc.103

Souza, A. S., Rerko, L., \& Oberauer, K. (2015). Refreshing memory traces: thinking of an item improves retrieval from visual working memory. Annals of the New York Academy of Sciences, 1339(1), 20-31. https://doi.org/10.1111/nyas.12603

Souza, A. S., Vergauwe, E., \& Oberauer, K. (2018). Where to attend next: guiding refreshing of visual, spatial, and verbal representations in working memory. Annals of the New York Academy of Sciences, 20-22. https://doi.org/10.1111/nyas.13621

Thalmann, M., Souza, A. S., \& Oberauer, K. (2019). Revisiting the attentional demands of rehearsal in working-memory tasks. Journal of Memory and Language, 105, 1-18.

Turley-Ames, K. J., \& Whitfield, M. M. (2003). Strategy training and working memory task performance. Journal of Memory and Language, 49(4), 446-468. https://doi.org/10.1016/S0749-596X(03)00095-0

Vergauwe, E., Camos, V., \& Barrouillet, P. (2014). The impact of storage on processing: How is information maintained in working memory? Journal of Experimental Psychology: Learning, Memory, and Cognition, 40(4), 1072.

Vergauwe, E., \& Langerock, N. (2017). Attentional refreshing of information in working memory: Increased immediate accessibility of just-refreshed representations. Journal of Memory and Language, 96, 23-35.

Vo, M. L. H., Conrad, M., Kuchinke, L., Urton, K., Hofmann, M. J., \& Jacobs, A. M. (2009). The Berlin affective word list reloaded (BAWL-R). Behavior Research Methods, 41(2), $534-538$ 